\title{
Kinematic Analysis of the 3-RPS Cube Parallel Manipulator
}

\author{
Latifah Nurahmi ${ }^{1}$, Josef Schadlbauer ${ }^{2}$, Stéphane Caro*1, Manfred Husty ${ }^{2}$, and Philippe Wenger ${ }^{1}$ \\ ${ }^{1}$ Institut de Recherche en Communications et Cybernétique de Nantes, France; e-mails: \\ \{latifah.nurahmi, stephane.caro, philippe.wenger\}@irccyn.ec-nantes.fr \\ ${ }^{2}$ University of Innsbruck, Austria; e-mails: \{josef.schadlbauer, manfred.husty\}@uibk.ac.at
}

\begin{abstract}
The 3-RPS Cube parallel manipulator, a three-degree-of-freedom parallel manipulator initially proposed by Huang et al. in 1995, is analysed in this paper with an algebraic approach, namely Study kinematic mapping of the Euclidean group SE(3) and is described by a set of eight constraint equations.

A primary decomposition is computed over the set of eight constraint equations and reveals that the manipulator has only one operation mode. Inside this operation mode, it turns out that the direct kinematics of the manipulator with arbitrary values of design parameters and joint variables, has sixteen solutions in the complex space. A geometric interpretation of the real solutions is given.

The singularity conditions are obtained by deriving the determinant of the Jacobian matrix of the eight constraint equations. All the singular poses are mapped onto the joint space and are geometrically interpreted. By parametrizing the set of constraint equations under the singularity conditions, it is shown that the manipulator is in actuation singularity. The uncontrolled motion gained by the moving platform is also provided.
\end{abstract}

*corresponding author, Paper JMR-14-1262, corresponding author's last name: CARO 
The motion of the moving platform is essentially determined by the fact that three vertices in the moving platform move in three mutually orthogonal planes. The workspace of each point of the moving platform (with exception of the three vertices) is bounded by a Steiner surface. This type of motion has been studied by Darboux in 1897.

Moreover, the 3-dof motion of the 3-RPS Cube parallel manipulator contains a special one-degree-of-freedom motion, called the Vertical Darboux Motion. In this motion, the moving platform can rotate and translate about and along the same axis simultaneously. The surface generated by a line in the moving platform turns out to be a right-conoid surface.

Keywords: 3-RPS-Cube, parallel manipulators, singularities, operation mode, motion type, Darboux motion.

\section{Introduction}

Since the development of robot technology, the lower-mobility parallel manipulators have been extensively studied. One parallel manipulator of the 3-dof family is the 3-RPS Cube and was proposed by Huang et al. in 1995 [1]. The 3-RPS Cube parallel manipulator, shown in Fig. 1, is composed of a cube-shaped base, an equilateral triangular-shaped platform, and three identical legs. Each leg is composed of a revolute joint, an actuated prismatic joint and a spherical joint mounted in series.

By referring to the design of the 3-RPS Cube manipulator, the type synthesis of 3 dof rotational manipulators with no intersecting axes was discussed in [2]. The kinematic characteristics of this mechanism were studied in [3-5], by identifying the principal screws, and the authors showed that the manipulator belongs to the general third-order screw system, which can rotate in three dimensions and the axes do not intersect.

In [6], Huang et al. showed that the mechanism is able to perform a motion along its diagonal, which is known as the Vertical Darboux Motion (VDM). Several mechanical generators of the VDM were later revealed by Lee and Hervé [7], in which one point in the moving platform is compelled to move in a plane. 
Later in [8], the authors showed that the manufacturing errors have little impact on the motion properties of the 3-RPS Cube parallel manipulator. By analysing the Instantaneous Screw Axes (ISA), Chen et al. showed in [9] that this mechanism performs parasitic motions, in which the translations and the rotations are coupled.

By using an algebraic description of the manipulator and the Study kinematic mapping, a characterisation of the operation mode, the direct kinematics, the general motion, and the singular poses of the 3-RPS Cube parallel manipulator are discussed in more detail in this paper, which is based on [10-14]. The derivation of the constraint equations is the first essential step to reveal the existence of only one operation mode and to solve the direct kinematics problem.

In 1897, Darboux [15] studied the 3-dof motion where the vertices of a triangle are compelled to remain in the planes of a trihedron respectively. The three planes are mutually orthogonal and this is the case of the 3-RPS Cube parallel manipulator. Darboux showed that in this 3-dof motion, the workspace of each point of the moving platform is bounded by a Steiner surface, while the vertices of the moving platform remain in the planes.

Under the condition that the prismatic lengths remain equal, the moving platform of the manipulator is able to perform the VDM. It follows from Bottema and Roth [15] that this motion is the result of a rotation about an axis and a harmonic translation along the same axis. In this motion, all points in the moving platform (except the geometric center of the moving platform) move in ellipses and the path of a line in the moving platform is a right-conoid surface.

The singularities are examined in this paper by deriving the determinant of the Jacobian matrix of the constraint equations with respect to the Study parameters. Based on the reciprocity conditions, Joshi and Tsai in [16] developed a procedure to express the Jacobian matrix $\mathbf{J}$ of lower-mobility parallel manipulators that comprises both actuation and constraint wrenches. In this paper, this matrix is named the extended Jacobian matrix $\left(\mathbf{J}_{E}\right)$ of the lower-mobility parallel manipulators, as explained in [17-21]. The rows of $\mathbf{J}_{E}$ are composed of $n$ linearly independent actuation wrenches plus $(6-n)$ linearly 
independent constraint wrenches.

In a general configuration, the constraint wrench system, $\mathcal{W}_{c}$, must be reciprocal to the twist system of the moving platform of the parallel manipulator. A constraint singularity occurs when the $(6-n)$ constraint wrench system $\mathcal{W}_{c}$ degenerates. In such a configuration, at least one of the initial constrained motions will no longer be constrained. As a result, the mechanism gains one or several dof. This can lead to a change in the motion pattern of the mechanism, which then can switch to another operation mode.

By locking the actuated joints of the parallel manipulator, the moving platform must be fully constrained, i.e., the system spanned by the actuation wrench system, $\mathcal{W}_{a}$, and constraint wrench system, $\mathcal{W}_{c}$, must span a 6 -system. An actuation singularity hence occurs when this overall wrench system of the manipulator degenerates, i.e., is not a 6-system any more, while the manipulator does not reach a constraint singularity.

This concept will be applied in this paper to illustrate the singularities of the extended Jacobian matrix $\left(\mathbf{J}_{E}\right)$ of the 3-RPS Cube parallel manipulator. It allows us to investigate the actuation and constraint singularities that occur during the manipulator motion.

This paper is organized as follows: A detailed description of the manipulator architecture is given in Section 2. The constraint equations of the manipulator are expressed in Section 3. These equations are used to identify the operation mode(s) and the solutions of the direct kinematics of the manipulator in Section 4. In Section 5, the conditions on the leg lengths for the manipulator to reach a singularity configuration are presented. Eventually, the general motion and the Vertical Darboux Motion (VDM) of the manipulator are reviewed in Sections 6 and 7.

\section{Manipulator Architecture}

The 3-RPS Cube parallel manipulator shown in Fig. 1, is composed of a cube-shaped base, an equilateral triangular-shaped platform and three identical legs. Each leg is composed of a revolute joint, an actuated prismatic joint and a spherical joint mounted in series. The origin $O$ of the fixed frame $\Sigma_{0}$ is shifted along $\sigma_{0}=\left[h_{0}, h_{0}, h_{0}\right]$ from the center of the base in order to fulfill the identity condition (when the fixed frame and the moving 
frame are coincident), as shown by the large and red dashed box in Fig. 1. Likewise, the origin $P$ of the moving frame $\Sigma_{1}$ is shifted along $\sigma_{1}=\left[h_{1}, h_{1}, h_{1}\right]$ as described by the small and blue dashed box in Fig. 1.

The revolute joint in the $i$-th $(i=1 \ldots 3)$ leg is located at point $A_{i}$, its axis being along vector $\mathbf{s}_{i}$, while the spherical joint is located at point $B_{i}$, the $i$-th corner of the moving platform. The distance between the origin $O$ of the fixed frame $\Sigma_{0}$ and point $A_{i}$ is equal to $h_{0} \sqrt{2}$. The axes $\mathbf{s}_{1}, \mathbf{s}_{2}$ and $\mathbf{s}_{3}$ are orthogonal to each other. The moving platform has an equilateral triangle shape and its circumradius is equal to $d_{1}=h_{1} \sqrt{6} / 3$.

Each pair of vertices $A_{i}$ and $B_{i}(i=1,2,3)$ is connected by a prismatic joint. The prismatic length is denoted by $r_{i}$. Since the $i$-th prismatic length is orthogonal to the revolute axis $\mathbf{s}_{i}$, the leg $A_{i} B_{i}$ moves in a plane normal to $\mathbf{s}_{i}$.

As a consequence, there are five parameters, namely $r_{1}, r_{2}, r_{3}, h_{0}$, and $h_{1} . h_{0}$ and $h_{1}$ are design parameters, while $r_{1}, r_{2}$, and $r_{3}$ are joint variables that determine the manipulator motion.

\section{Constraint Equations}

In this section, the constraint equations are expressed whose solutions illustrate the possible poses of the moving platform (coordinate frame $\Sigma_{1}$ ) with respect to $\Sigma_{0}$. In the following, we use projective coordinates to define the position vectors of points $A_{i}$ and $B_{i}$. The coordinates of points $A_{i}$ and points $B_{i}$ expressed in $\Sigma_{0}$ and $\Sigma_{1}$ are respectively:

$$
\begin{array}{ll}
\mathbf{r}_{A_{1}}^{0}=\left[1,0,-h_{0},-h_{0}\right]^{T}, & \mathbf{r}_{B_{1}}^{1}=\left[1,0,-h_{1},-h_{1}\right]^{T}, \\
\mathbf{r}_{A_{2}}^{0}=\left[1,-h_{0}, 0,-h_{0}\right]^{T}, & \mathbf{r}_{B_{2}}^{1}=\left[1,-h_{1}, 0,-h_{1}\right]^{T}, \\
\mathbf{r}_{A_{3}}^{0}=\left[1,-h_{0},-h_{0}, 0\right]^{T}, & \mathbf{r}_{B_{3}}^{1}=\left[1,-h_{1},-h_{1}, 0\right]^{T}
\end{array}
$$

To obtain the coordinates of points $B_{1}, B_{2}$ and $B_{3}$ expressed in $\Sigma_{0}$, the Study parametrization of a spatial Euclidean transformation matrix $\mathbf{M} \in S E(3)$ is used as follows: 


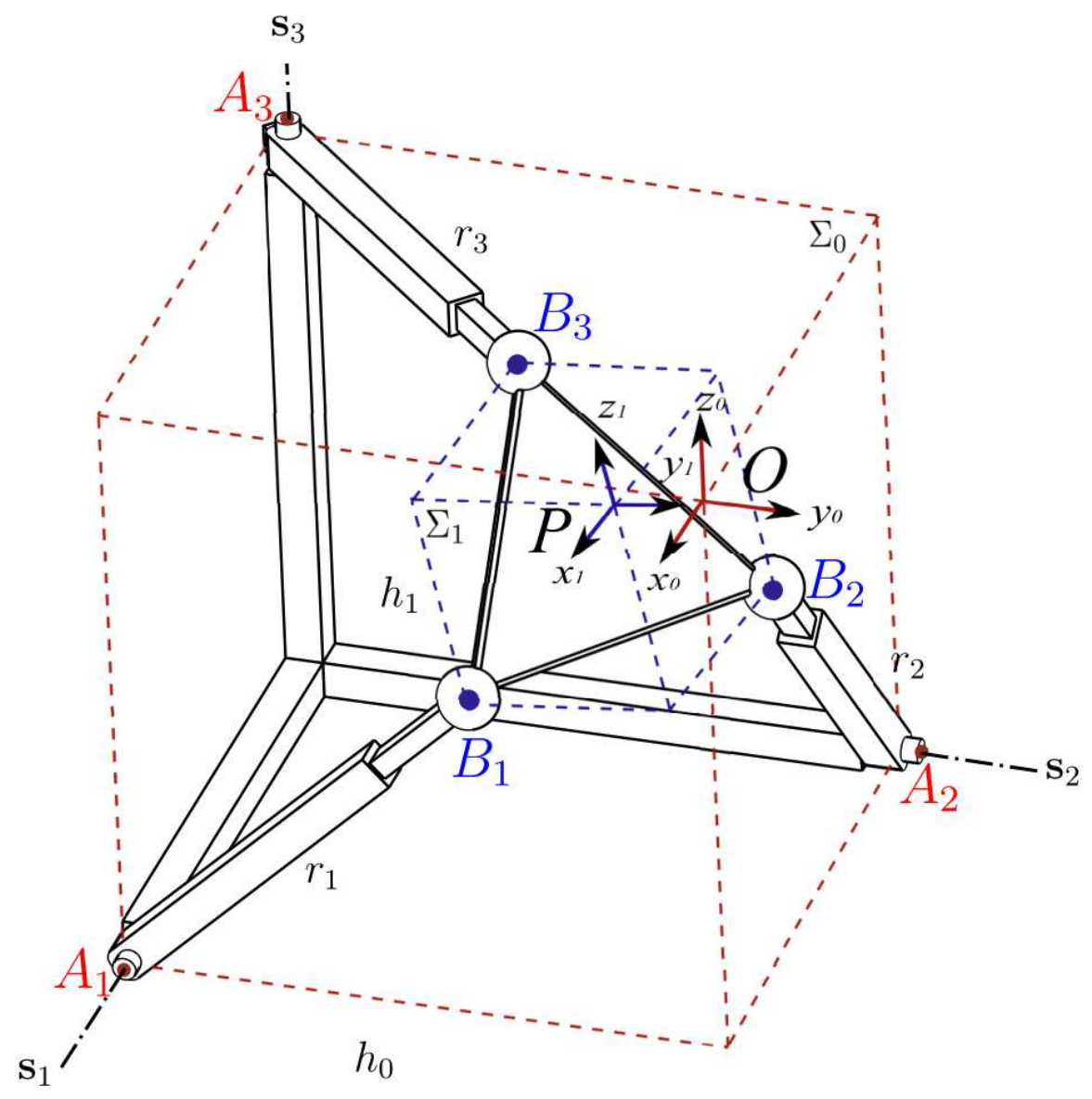

Figure 1: The 3-RPS Cube Parallel Manipulator.

$$
\mathbf{M}=\left(\begin{array}{cc}
x_{0}^{2}+x_{1}^{2}+x_{2}^{2}+x_{3}^{2} & \mathbf{0}_{3 \times 1}^{T} \\
\mathbf{M}_{T} & \mathbf{M}_{R}
\end{array}\right)
$$

where $\mathbf{M}_{T}$ and $\mathbf{M}_{R}$ represent the translational and rotational parts of transformation matrix $\mathbf{M}$, respectively, and are expressed as follows: 


$$
\begin{gathered}
\mathbf{M}_{T}=\left(\begin{array}{c}
2\left(-x_{0} y_{1}+x_{1} y_{0}-x_{2} y_{3}+x_{3} y_{2}\right) \\
2\left(-x_{0} y_{2}+x_{1} y_{3}+x_{2} y_{0}-x_{3} y_{1}\right) \\
2\left(-x_{0} y_{3}-x_{1} y_{2}+x_{2} y_{1}+x_{3} y_{0}\right)
\end{array}\right), \\
\mathbf{M}_{R}=\left(\begin{array}{ccc}
x_{0}^{2}+x_{1}^{2}-x_{2}^{2}-x_{3}^{2} & 2\left(x_{1} x_{2}-x_{0} x_{3}\right) & 2\left(x_{1} x_{3}+x_{0} x_{2}\right) \\
2\left(x_{1} x_{2}+x_{0} x_{3}\right) & x_{0}^{2}-x_{1}^{2}+x_{2}^{2}-x_{3}^{2} & 2\left(x_{2} x_{3}-x_{0} x_{1}\right) \\
2\left(x_{1} x_{3}-x_{0} x_{2}\right) & 2\left(x_{2} x_{3}+x_{0} x_{1}\right) & x_{0}^{2}-x_{1}^{2}-x_{2}^{2}+x_{3}^{2}
\end{array}\right)
\end{gathered}
$$

The parameters $x_{0}, x_{1}, x_{2}, x_{3}, y_{0}, y_{1}, y_{2}, y_{3}$, which appear in matrix $\mathbf{M}$, are called Study parameters. These parameters make it possible to parametrize $S E(3)$ with dual quaternions. The Study kinematic mapping maps each spatial Euclidean displacement of $S E(3)$ via transformation matrix $\mathbf{M}$ onto a projective point $X\left[x_{0}: x_{1}: x_{2}: x_{3}: y_{0}: y_{1}: y_{2}: y_{3}\right]$ in the 6-dimensional Study quadric $S \in \mathbb{P}^{7}[14]$, such that:

$$
\begin{gathered}
S E(3) \rightarrow X \in \mathbb{P}^{7} \\
\left(x_{0}: x_{1}: x_{2}: x_{3}: y_{0}: y_{1}: y_{2}: y_{3}\right)^{T} \neq(0: 0: 0: 0: 0: 0: 0: 0)^{T}
\end{gathered}
$$

Every projective point $X$ will represent a spatial Euclidean displacement, if it fulfills the following equation and inequality:

$$
\begin{gathered}
x_{0} y_{0}+x_{1} y_{1}+x_{2} y_{2}+x_{3} y_{3}=0, \\
x_{0}^{2}+x_{1}^{2}+x_{2}^{2}+x_{3}^{2} \neq 0
\end{gathered}
$$

Those two conditions will be used in the following computations to simplify the algebraic expressions. The coordinates of points $B_{i}$ expressed in $\Sigma_{0}$ are obtained by:

$$
\mathbf{r}_{B_{i}}^{0}=\mathbf{M} \mathbf{r}_{B_{i}}^{1} \quad i=0, \ldots, 3
$$

As the coordinates of all points are given in terms of Study parameters, design parameters and joint variables, the constraint equations can be obtained by examining the 
manipulator architecture. The leg connecting points $A_{i}$ and $B_{i}$ is orthogonal to the axis $\mathbf{s}_{i}$ of the $i$-th revolute joint, expressed as follows:

$$
\begin{aligned}
& \mathbf{s}_{1}=[0,1,0,0]^{T} \\
& \mathbf{s}_{2}=[0,0,1,0]^{T} \\
& \mathbf{s}_{3}=[0,0,0,1]^{T}
\end{aligned}
$$

Accordingly, the scalar product of vector $\left(\mathbf{r}_{B_{i}}^{0}-\mathbf{r}_{A_{i}}^{0}\right)$ and vector $\mathbf{s}_{i}$ vanishes, namely:

$$
\left(\mathbf{r}_{B_{i}}^{0}-\mathbf{r}_{A_{i}}^{0}\right)^{T} \mathbf{s}_{i}=0
$$

After computing the corresponding scalar products and removing the common denominators $\left(x_{0}^{2}+x_{1}^{2}+x_{2}^{2}+x_{3}^{2}\right)$, the following three equations come out:

$$
\begin{aligned}
& g_{1}:-h_{1} x_{0} x_{2}+h_{1} x_{0} x_{3}-h_{1} x_{1} x_{2}-h_{1} x_{1} x_{3}-x_{0} y_{1}+x_{1} y_{0}-x_{2} y_{3}+x_{3} y_{2}=0 \\
& g_{2}: h_{1} x_{0} x_{1}-h_{1} x_{0} x_{3}-h_{1} x_{1} x_{2}-h_{1} x_{2} x_{3}-x_{0} y_{2}+x_{1} y_{3}+x_{2} y_{0}-x_{3} y_{1}=0 \\
& g_{3}:-h_{1} x_{0} x_{1}+h_{1} x_{0} x_{2}-h_{1} x_{1} x_{3}-h_{1} x_{2} x_{3}-x_{0} y_{3}-x_{1} y_{2}+x_{2} y_{1}+x_{3} y_{0}=0
\end{aligned}
$$

To derive the constraint equations corresponding to the leg lengths, the joint variables $r_{i}$ are given and we assume that the distance between points $A_{i}$ and $B_{i}$ is constant, i.e. $r_{i}=$ const. It follows that point $B_{i}$ has the freedom to move along a circle of center $A_{i}$ and the distance equation can be formulated as $\left\|\left(\mathbf{r}_{B_{i}}^{0}-\mathbf{r}_{A_{i}}^{0}\right)\right\|^{2}=r_{i}^{2}$. As a consequence, the following three equations are obtained: 


$$
\begin{aligned}
& g_{4}: 2 h_{0}^{2} x_{0}^{2}+2 h_{0}^{2} x_{1}^{2}+2 h_{0}^{2} x_{2}^{2}+2 h_{0}^{2} x_{3}^{2}-4 h_{0} h_{1} x_{0}^{2}+4 h_{0} h_{1} x_{1}^{2}+2 h_{1}^{2} x_{0}^{2} \\
& +2 h_{1}^{2} x_{1}^{2}+2 h_{1}^{2} x_{2}^{2}+2 h_{1}^{2} x_{3}^{2}-8 h_{0} h_{1} x_{2} x_{3}-r_{1}^{2} x_{0}^{2}-r_{1}^{2} x_{1}^{2}-r_{1}^{2} x_{2}^{2} \\
& -r_{1}^{2} x_{3}^{2}-4 h_{0} x_{0} y_{2}-4 h_{0} x_{0} y_{3}-4 h_{0} x_{1} y_{2}+4 h_{0} x_{1} y_{3}+4 h_{0} x_{2} y_{0} \\
& +4 h_{0} x_{2} y_{1}+4 h_{0} x_{3} y_{0}-4 h_{0} x_{3} y_{1}+4 h_{1} x_{0} y_{2}+4 h_{1} x_{0} y_{3}+4 y_{0}^{2} \\
& -4 h_{1} x_{1} y_{2}+4 h_{1} x_{1} y_{3}-4 h_{1} x_{2} y_{0}+4 h_{1} x_{2} y_{1}-4 h_{1} x_{3} y_{0}+4 y_{1}^{2} \\
& -4 h_{1} x_{3} y_{1}+4 y_{2}^{2}+4 y_{3}^{2}=0 \\
& g_{5}: 2 h_{0}^{2} x_{0}^{2}+2 h_{0}^{2} x_{1}^{2}+2 h_{0}^{2} x_{2}^{2}+2 h_{0}^{2} x_{3}^{2}-4 h_{0} h_{1} x_{0}^{2}+4 h_{0} h_{1} x_{2}^{2}+2 h_{1}^{2} x_{0}^{2} \\
& +2 h_{1}^{2} x_{1}^{2}+2 h_{1}^{2} x_{2}^{2}+2 h_{1}^{2} x_{3}^{2}-8 h_{0} h_{1} x_{1} x_{3}-r_{2}^{2} x_{0}^{2}-r_{2}^{2} x_{1}^{2}-r_{2}^{2} x_{2}^{2} \\
& -r_{2}^{2} x_{3}^{2}-4 h_{0} x_{0} y_{1}-4 h_{0} x_{0} y_{3}+4 h_{0} x_{1} y_{0}-4 h_{0} x_{1} y_{2}+4 h_{0} x_{2} y_{1} \\
& -4 h_{0} x_{2} y_{3}+4 h_{0} x_{3} y_{0}+4 h_{0} x_{3} y_{2}+4 h_{1} x_{0} y_{1}+4 h_{1} x_{0} y_{3}+4 y_{0}^{2} \\
& -4 h_{1} x_{1} y_{0}-4 h_{1} x_{1} y_{2}+4 h_{1} x_{2} y_{1}-4 h_{1} x_{2} y_{3}-4 h_{1} x_{3} y_{0}+4 y_{1}^{2} \\
& +4 h_{1} x_{3} y_{2}+4 y_{2}^{2}+4 y_{3}^{2}=0 \\
& g_{6}: 2 h_{0}^{2} x_{0}^{2}+2 h_{0}^{2} x_{1}^{2}+2 h_{0}^{2} x_{2}^{2}+2 h_{0}^{2} x_{3}^{2}-4 h_{0} h_{1} x_{0}^{2}+4 h_{0} h_{1} x_{3}^{2}+2 h_{1}^{2} x_{0}^{2} \\
& +2 h_{1}^{2} x_{1}^{2}+2 h_{1}^{2} x_{2}^{2}+2 h_{1}^{2} x_{3}^{2}-8 h_{0} h_{1} x_{1} x_{2}-r_{3}^{2} x_{0}^{2}-r_{3}^{2} x_{1}^{2}-r_{3}^{2} x_{2}^{2} \\
& -r_{3}^{2} x_{3}^{2}-4 h_{0} x_{0} y_{1}-4 h_{0} x_{0} y_{2}+4 h_{0} x_{1} y_{0}+4 h_{0} x_{1} y_{3}+4 h_{0} x_{2} y_{0} \\
& -4 h_{0} x_{2} y_{3}-4 h_{0} x_{3} y_{1}+4 h_{0} x_{3} y_{2}+4 h_{1} x_{0} y_{1}+4 h_{1} x_{0} y_{2}+4 y_{0}^{2} \\
& -4 h_{1} x_{1} y_{0}+4 h_{1} x_{1} y_{3}-4 h_{1} x_{2} y_{0}-4 h_{1} x_{2} y_{3}-4 h_{1} x_{3} y_{1}+4 y_{1}^{2} \\
& +4 h_{1} x_{3} y_{2}+4 y_{2}^{2}+4 y_{3}^{2}=0
\end{aligned}
$$

The Study equation in Eq. (5) is added since all solutions have to be within the Study quadric, i.e.:

$$
g_{7}: x_{0} y_{0}+x_{1} y_{1}+x_{2} y_{2}+x_{3} y_{3}=0
$$

Under the condition $\left(x_{0}^{2}+x_{1}^{2}+x_{2}^{2}+x_{3}^{2} \neq 0\right)$, we can find all possible points in $\mathbb{P}^{7}$ that satisfy those seven equations. To exclude the exceptional generator $\left(x_{0}=x_{1}=x_{2}=x_{3}=\right.$ $0)$, we add the following normalization equation: 


$$
g_{8}: x_{0}^{2}+x_{1}^{2}+x_{2}^{2}+x_{3}^{2}-1=0
$$

It assures that there is no point of the exceptional generator that appears as a solution. However, for each projective solution point, we obtain two affine representatives. This has to be taken into account for the enumeration of the number of solutions.

\section{Solving the System}

Solving the direct kinematics means finding all possible points in $\mathbb{P}^{7}$ that fulfill the set of equations $\left\{g_{1}, \ldots, g_{8}\right\}$. Those points are the solutions of the eight constraint equations that represent all feasible poses of the 3-RPS Cube parallel manipulator. They also depend on the design parameters $\left(h_{0}, h_{1}\right)$ and the joint variables $\left(r_{1}, r_{2}, r_{3}\right)$.

The set of eight constraint equations are always written as a polynomial ideal with variables $\left\{x_{0}, x_{1}, x_{2}, x_{3}, y_{0}, y_{1}, y_{2}, y_{3}\right\}$ over the coefficient ring $\mathbb{C}\left[h_{0}, h_{1}, r_{1}, r_{2}, r_{3}\right]$. Although the solutions of the direct kinematics can be complex, they are still considered as solutions. To apply the method of algebraic geometry, the ideal is now defined as:

$$
\mathcal{I}=<g_{1}, g_{2}, g_{3}, g_{4}, g_{5}, g_{6}, g_{7}, g_{8}>
$$

The vanishing set $\mathbf{V}(\mathcal{I})$ of the ideal $\mathcal{I}$ comprises all points in $\mathbb{P}^{7}$ for which all equations vanish, namely all solutions of the direct kinematic problem. At this point, the following ideal is examined, which is independent of the joints variables $r_{1}, r_{2}$ and $r_{3}$ :

$$
\mathcal{J}=<g_{1}, g_{2}, g_{3}, g_{7}>
$$

The primary decomposition is computed to verify if the ideal $\mathcal{J}$ is the intersection of several smaller ideals. The primary decomposition returns several $\mathcal{J}_{i}$ in which $\mathcal{J}=\bigcap_{i} \mathcal{J}_{i}$. In other words, the vanishing set is given by $\mathbf{V}(\mathcal{J})=\bigcup_{i} \mathbf{V}\left(\mathcal{J}_{i}\right)$. It expresses that the variety $\mathbf{V}(\mathcal{J})$ is the union of some other or simpler varieties $\mathbf{V}\left(\mathcal{J}_{i}\right)$.

The primary decomposition geometrically tells us that the intersection of those equa- 
tions will split into smaller parts. Indeed, it turns out that the ideal $\mathcal{J}$ is decomposed into two components $\mathcal{J}_{i}$ as:

$$
\mathcal{J}=\bigcap_{i=1}^{2} \mathcal{J}_{i}
$$

with the results of primary decompositions ${ }^{1}$ as follows:

$$
\begin{aligned}
& \mathcal{J}_{1}=<x_{0} y_{0}+x_{1} y_{1}+x_{2} y_{2}+x_{3} y_{3}, \ldots> \\
& \mathcal{J}_{2}=<x_{0}, x_{1}, x_{2}, x_{3}>
\end{aligned}
$$

An inspection of the vanishing set $\mathbf{V}\left(\mathcal{J}_{2} \cup g_{8}\right)$ yields an empty result, since the set of polynomials $\left\{x_{0}, x_{1}, x_{2}, x_{3}, x_{0}^{2}+x_{1}^{2}+x_{2}^{2}+x_{3}^{2}-1=0\right\}$ can never vanish simultaneously over $\mathbb{R}$ or $\mathbb{C}$. Therefore, only one component is left and as a consequence, the manipulator has only one operation mode, which is defined by $\mathcal{J}_{1}$. To complete the analysis, the remaining equations have to be added by writing:

$$
\mathcal{K}_{i}=\mathcal{J}_{i} \cup<g_{4}, g_{5}, g_{6}, g_{8}>
$$

Since there is only one component, the vanishing set of $\mathcal{I}$ is now defined by:

$$
\mathbf{V}(\mathcal{I})=\mathbf{V}\left(\mathcal{K}_{1}\right)
$$

From the primary decomposition, it is shown that the ideal $\mathcal{I}$ cannot be split and $\mathcal{K}_{1}$ is named $\mathcal{I}$ hereafter.

\subsection{Solutions for arbitrary design parameters}

The 3-RPS Cube manipulator generally has only one operation mode, which is described by the ideal $\mathcal{I}$. The solutions of the direct kinematic problem in this operation mode will be given for arbitrary values of design parameters $\left(h_{0}, h_{1}\right)$. To find out the Hilbert dimension of the ideal $\mathcal{I}$, the certain values of design parameters are chosen as $h_{0}=2 \mathrm{~m}$

\footnotetext{
${ }^{1}$ For complete results of the primary decomposition, the reader may refer to: http://www.irccyn.ec-nantes.fr/ caro/ASME_JMR/JMR_14_1262/Appendix_3RPSCube.pdf
} 
and $h_{1}=1 \mathrm{~m}$. It turns out that:

$$
\operatorname{dim}(\mathcal{I})=0
$$

The $\operatorname{dim}$ denotes the dimension over $\mathbb{C}\left[h_{0}, h_{1}, r_{1}, r_{2}, r_{3}\right]$ and shows that the number of solutions to the direct kinematic problems is finite in this general mode. The number of solutions and the solutions themselves were computed via an ordered Gröbner basis, which led to a univariate polynomial of degree 32 . As two solutions of a system describe the same pose (position and orientation) of the moving platform, the number of solutions has to be halved into 16 solutions.

$$
|\mathbf{V}(\mathcal{I})|=16
$$

Therefore, there are at most 16 different solutions for given general design parameters and joint variables, i.e., there are theoretically 16 feasible poses of the moving platform for given joint variables. Notably, for arbitrarily values of design parameters and joint variables, some solutions might be complex.

\subsection{Solutions for equal leg lengths}

In the following subsection, it is assumed that all legs have the same length. The corresponding prismatic lengths are $r_{1}=r_{2}=r_{3}=r$. Similar computations can be performed which were done in the previous subsection to enumerate the Hilbert dimension of the ideal. The Hilbert dimension is calculated and it follows that:

$$
\operatorname{dim}(\mathcal{I})=0
$$

This shows that the solutions of the direct kinematics problem with equal leg lengths are finite. When the number of solutions is computed for the system, it has to be halved and the following result is obtained: 


$$
|\mathbf{V}(\mathcal{I})|=16
$$

The number of solutions for equal leg lengths is the same number as the solutions for arbitrary design parameters. Due to the fact that there are fewer parameters, the Gröebner basis can be computed without specifying any value. The solutions of Study parameters in the case of equal leg lengths are $x_{1}=x_{2}=x_{3}$ and $y_{1}=y_{2}=y_{3}$. One manipulator pose with equal prismatic lengths leads to the following solutions of Study parameters:

$$
\begin{aligned}
& x_{0}=\frac{1}{2} \frac{\sqrt{-h_{1}\left(\sqrt{3 h_{0}^{2}-2 h_{1}^{2}+3 r^{2}}-3 h_{0}-2 h_{1}\right)}}{h 1} \\
& x_{1}=\frac{1}{6} \frac{\sqrt{3} \sqrt{h_{1}\left(\sqrt{3 h_{0}^{2}-2 h_{1}^{2}+3 r^{2}}-3 h_{0}+2 h_{1}\right)}}{h 1} \\
& y_{0}=\frac{1}{12} \frac{\sqrt{3}\left(h_{1}\left(\sqrt{3 h_{0}^{2}-2 h_{1}^{2}+3 r^{2}}-3 h_{0}+2 h_{1}\right)\right)^{3 / 2}}{h 1^{2}} \\
& y_{1}=-\frac{1}{12 h_{1}} \sqrt{-h_{1}\left(\sqrt{3 h_{0}^{2}-2 h_{1}^{2}+3 r^{2}}-3 h_{0}-2 h_{1}\right)}\left(2 h_{1}\right. \\
& x_{1}=x_{2}=x_{3} \\
& y_{1}=y_{2}=y_{3}+\sqrt{\left.3 h_{0}^{2}-2 h_{1}^{2}+3 r^{2}\right)}
\end{aligned}
$$

\subsection{Operation mode analysis}

In the previous section, the joint variables $\left(r_{1}, r_{2}, r_{3}\right)$ were fixed. In this section, they can change, i.e., the behaviour of the mechanism is studied when the prismatic joints are actuated. The joint variables $\left(r_{1}, r_{2}\right.$ and $\left.r_{3}\right)$ are used as unknowns and the computation of the Hilbert dimension shows that:

$$
\overline{\operatorname{dim}}(\mathcal{I})=3
$$


where $\overline{\operatorname{dim}}$ denotes the dimension over $\mathbb{C}\left[h_{0}, h_{1}\right]$ and shows that the manipulator has 3 dof in general motion.

The matrix $\mathbf{M} \in S E(3)$ in Eq. (2) represents a discrete screw motion from the pose corresponding to the identity condition, where $\Sigma_{0}$ and $\Sigma_{1}$ are coincident, to the transformed pose of $\Sigma_{1}$ with respect to $\Sigma_{0}$. A discrete screw motion is the concatenation of a rotation about an axis and a translation along the same axis. The axis $\mathcal{A}$, the translational distance $s$, and the rotational angle $\varphi$ of the discrete screw motion can be computed from the matrix M. This information can also be obtained directly from the Study parameters, as they contain the information on the transformation. The Plücker coordinates $L=\left(p_{0}: p_{1}: p_{2}: p_{3}: p_{4}: p_{5}\right)$ of the corresponding discrete screw motion are expressed as:

$$
\begin{aligned}
& p_{0}=\left(-x_{1}^{2}-x_{2}^{2}-x_{3}^{2}\right) x_{1}, \\
& p_{1}=\left(-x_{1}^{2}-x_{2}^{2}-x_{3}^{2}\right) x_{2}, \\
& p_{2}=\left(-x_{1}^{2}-x_{2}^{2}-x_{3}^{2}\right) x_{3}, \\
& p_{3}=x_{0} y_{0} x_{1}-\left(-x_{1}^{2}-x_{2}^{2}-x_{3}^{2}\right) y_{1}, \\
& p_{4}=x_{0} y_{0} x_{2}-\left(-x_{1}^{2}-x_{2}^{2}-x_{3}^{2}\right) y_{2}, \\
& p_{5}=x_{0} y_{0} x_{3}-\left(-x_{1}^{2}-x_{2}^{2}-x_{3}^{2}\right) y_{3} .
\end{aligned}
$$

The unit vector of an axis $\mathcal{A}$ of the corresponding discrete screw motion is given by $\left[p_{0}, p_{1}, p_{2}\right]^{T}$. The Plücker coordinates of a line should satisfy the following condition [22]:

$$
p_{0} p_{3}+p_{1} p_{4}+p_{2} p_{5}=0
$$

The rotational angle $\varphi$ can be enumerated directly from $\cos \left(\frac{\varphi}{2}\right)=x_{0}$, whereas the translational distance $s$ of the transformation can be computed from the Study parameters, as follows: 


$$
s=\frac{2 y_{0}}{\sqrt{x_{1}^{2}+x_{2}^{2}+x_{3}^{2}}}
$$

The following example shows the manipulator poses by solving the direct kinematic problem. Arbitrary values are assigned to the design parameters and joint variables as follows: $h_{0}=2 \mathrm{~m}, h_{1}=1 \mathrm{~m}, r_{1}=1.2 \mathrm{~m}, r_{2}=2 \mathrm{~m}$, and $r_{3}=1.5 \mathrm{~m}$. By considering only the real solutions, the manipulator has two solutions for those design parameters and joint variables.

The first solution of the direct kinematics is depicted in Fig. 2(a), with $\left(x_{0}: x_{1}: x_{2}\right.$ : $\left.x_{3}: y_{0}: y_{1}: y_{2}: y_{3}\right)=(-0.961:-0.189,-0.153: 0.128:-0.007: 0.304:-0.250: 0.089)$. The discrete screw motion of the moving platform from identity into the actual pose in Fig. 2(a) is along the axis $\mathcal{A}_{1}$. In Plücker coordinates, it is given by $\left(p_{0}: p_{1}: p_{2}: p_{3}:\right.$ $\left.p_{4}: p_{5}\right)=(0.014: 0.011:-0.009: 0.021:-0.020: 0.007)$. The rotational angle and translational distance along the screw axis $\mathcal{A}_{1}$ are $\varphi_{1}=5.725 \mathrm{rad}$ and $s_{1}=-0.057 \mathrm{~m}$, respectively.

Figure 2(b) illustrates the second solution of the direct kinematic problem, with $\left(x_{0}\right.$ : $\left.x_{1}: x_{2}: x_{3}: y_{0}: y_{1}: y_{2}: y_{3}\right)=(0.962: 0.056:-0.021:-0.265: 0.001:-0.293: 0.232:$ $-0.076)$. The moving platform is transformed from the identity into the final pose via the axis $\mathcal{A}_{2}$ in Fig. 2(b), with rotational angle $\varphi_{2}=0.552 \mathrm{rad}$ and translational distance $s_{2}=0.01 \mathrm{~m}$. The Plücker coordinate vector of the discrete screw motion is defined by $\left(p_{0}: p_{1}: p_{2}: p_{3}: p_{4}: p_{5}\right)=(-0.004: 0.001: 0.019:-0.021: 0.017:-0.006)$.

\section{Singularity Conditions of the Manipulator}

The manipulator reaches a singular configuration when the determinant of the Jacobian matrix vanishes. The Jacobian matrix is the matrix of all first order partial derivatives of eight constraint equations $\left\{g_{1}, g_{2}, g_{3}, g_{4}, g_{5}, g_{6}, g_{7}, g_{8}\right\}$ with respect to $\left\{x_{0}, x_{1}, x_{2}, x_{3}, y_{0}, y_{1}, y_{2}, y_{3}\right\}$. Since the manipulator has one operation mode, the singular configurations occur within this operation mode only. In the kinematic image space, the singular poses are computed by taking the Jacobian matrix from $\mathcal{I}$ : 


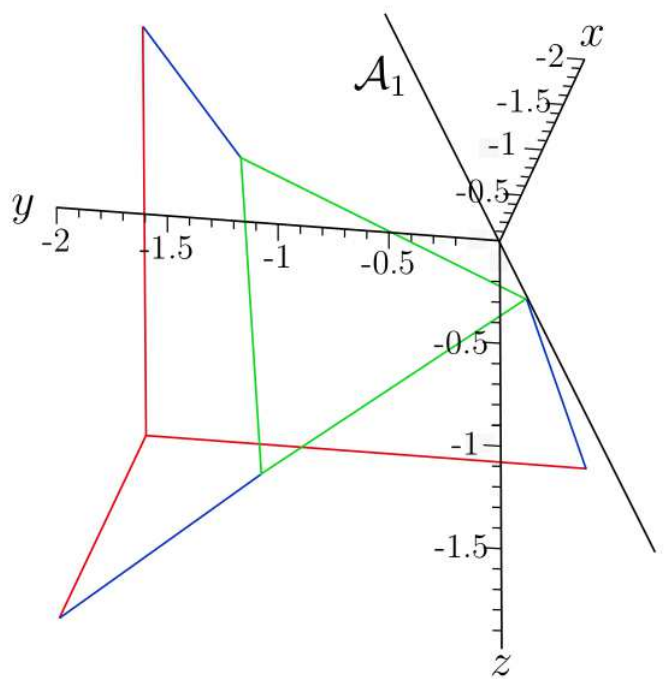

(a)

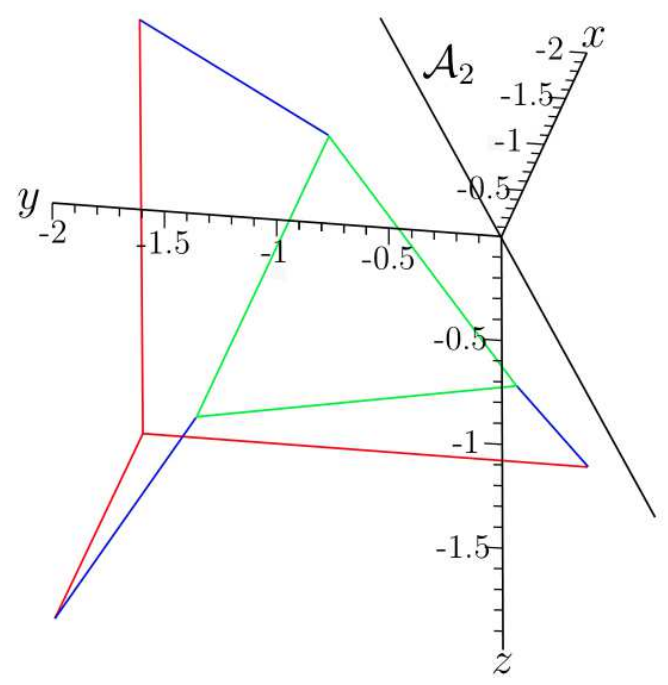

(b)

Figure 2: Solutions of the Direct Kinematics.

$$
\mathbf{J}=\left(\frac{\partial g_{j}}{\partial x_{k}}, \frac{\partial g_{j}}{\partial y_{k}}\right), \quad j=1, \ldots, 8, k=0, \ldots, 3
$$

The vanishing condition $\operatorname{det}(\mathbf{J})=0$ of the determinant $\mathbf{J}$ is denoted by $\mathcal{S}$. The factorization of the equation of the Jacobian determinant splits it into two components, namely $\mathcal{S}_{1}: \operatorname{det}_{1}(\mathbf{J})=0$ and $\mathcal{S}_{2}: \operatorname{det}_{2}(\mathbf{J})=0$.

$$
\begin{gathered}
\operatorname{det}(\mathbf{J})=0 \\
\operatorname{det}_{1}(\mathbf{J}) \operatorname{det}_{2}(\mathbf{J})=0
\end{gathered}
$$

It shows that the overall determinant will vanish if either $\mathcal{S}_{1}$ or $\mathcal{S}_{2}$ vanishes or both $\mathcal{S}_{1}$ and $\mathcal{S}_{2}$ vanish simultaneously. By adding the expression of the Jacobian determinant into the system $\mathcal{I}$, the new ideal associated with the singular poses can be defined as:

$$
\mathcal{L}_{i}=\mathcal{I} \cup \mathcal{S}_{i} \quad i=1,2
$$

The ideals now consist of a set of nine equations $\mathcal{L}_{i}=<g_{1}, g_{2}, g_{3}, g_{4}, g_{5}, g_{6}, g_{7}, g_{8}, g_{9}>$. The ninth equation is the determinant of the Jacobian matrix. 
In mechanics, the singularity surface is desirable also in the joint space $\mathcal{R}$, where $\mathcal{R}$ is the polynomial ring over variables $r_{1}, r_{2}$ and $r_{3}$. To obtain the singularity surface in $\mathcal{R}$, the following projections are determined from ideals $\mathcal{L}_{i}$ :

$$
\mathcal{L}_{i} \rightarrow \mathcal{R} \quad i=1,2
$$

Algebraically, each projection is an elimination of Study parameters from the ideal $\mathcal{L}_{i}$ and is mapped onto one equation generated by $r_{1}, r_{2}$ and $r_{3}$. It was not possible to compute the elimination in general, thus we assigned some values to the design parameters, namely $h_{0}=2 \mathrm{~m}$ and $h_{1}=1 \mathrm{~m}$. The eight Study parameters $x_{0}, x_{1}, x_{2}, x_{3}, y_{0}, y_{1}, y_{2}, y_{3}$ were eliminated to obtain a single polynomial in $r_{1}, r_{2}$ and $r_{3}$.

For the system $\mathcal{L}_{1}$, the elimination yields a polynomial of degree four in $r_{1}, r_{2}$ and $r_{3}$ in Eq. (32) and its zero set of polynomial is plotted in Fig. 3. By taking a point on this surface, we are able to compute the direct kinematics of at least one singularity pose.

$$
r_{1}^{4}-r_{1}^{2} r_{2}^{2}+r_{2}^{4}-r_{1}^{2} r_{3}^{2}-r_{2}^{2} r_{3}^{2}+r_{3}^{4}-2 r_{1}^{2}-2 r_{2}^{2}-2 r_{3}^{2}-20=0
$$

Due to the heavy elimination process of Study parameters from ideal $\mathcal{L}_{2}$, some arbitrary values have been assigned to the joint variables $r_{1}=2 \mathrm{~m}$ and $r_{2}=1.7 \mathrm{~m}$. Then the elimination can be carried out and the result is a univariate polynomial of degree 64 in $r_{3}$.

Let us consider one singularity configuration of the manipulator when the moving frame $\Sigma_{1}$ coincides with the fixed frame $\Sigma_{0}$ and all joint variables have the same values. The system $\mathcal{L}_{2}$ is now solved by assigning the joint variables as $r_{1}=r_{2}=r_{3}$. The elimination process returns a univariate polynomial of degree 24 in $r_{3}$. The real solutions of joint variables in this condition is $r_{1}=r_{2}=r_{3}=\sqrt{2} \mathrm{~m}$.

The coordinates of points $B_{1}, B_{2}$ and $B_{3}$ can be determined by solving the direct kinematics. Accordingly, we can form the extended Jacobian matrix $\left(\mathbf{J}_{E}\right)$ of the manipulator, which is based on the screw theory. The rows of the extended Jacobian matrix $\mathbf{J}_{E}$ are composed of $n$ actuation wrenches $\mathcal{W}_{a}$ and $(6-n)$ constraint wrenches $\mathcal{W}_{c}$. Since the 


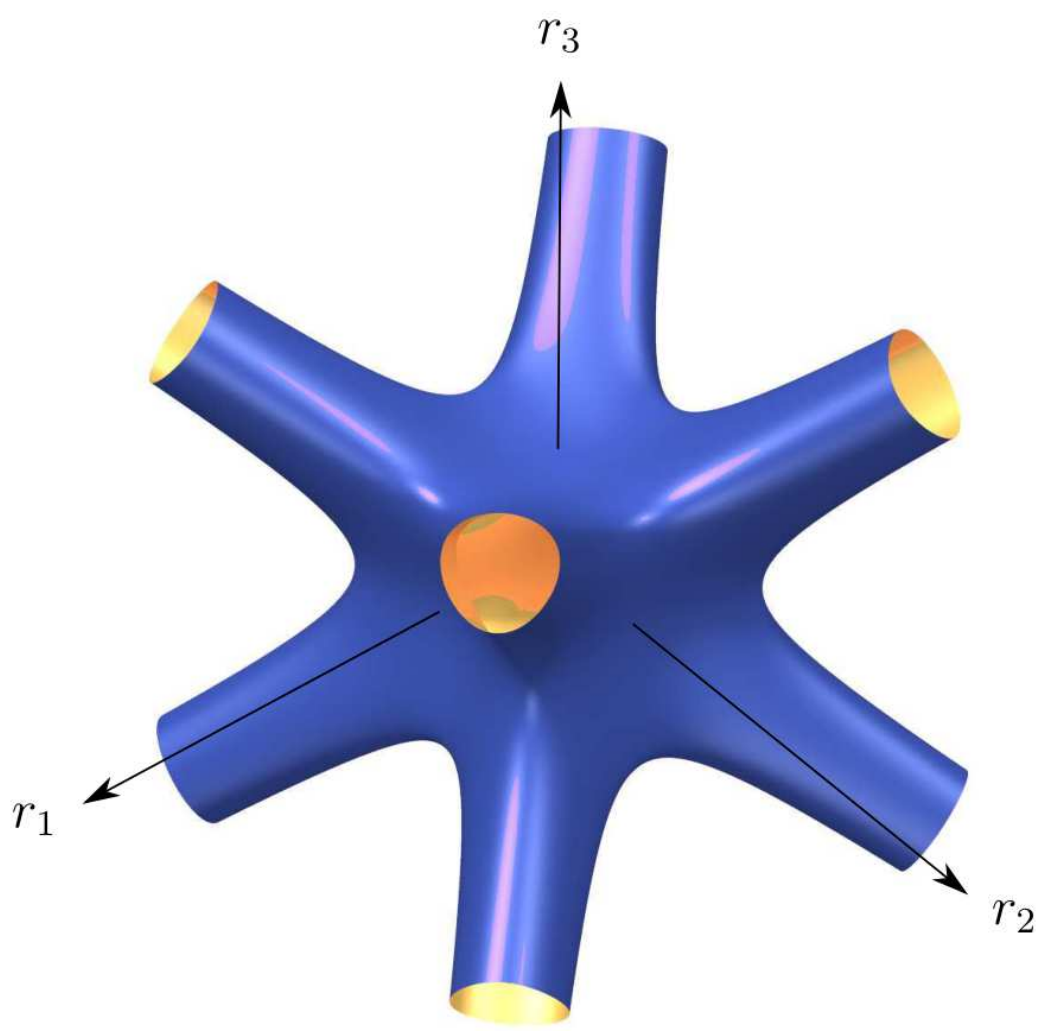

Figure 3: Singularity Surface of $\mathcal{L}_{1}$.

manipulator has 3 dof $(n=3)$, the first three rows of $\mathbf{J}_{E}$ comprise actuation wrenches and the last three rows are constraint wrenches.

By considering that the prismatic joints are actuated, each leg applies one actuation force whose axis is along the direction of the corresponding actuated joint $\mathbf{u}_{i}$, as follows:

$$
\begin{gathered}
\mathcal{F}_{a 1}=\left[\mathbf{u}_{1}, \mathbf{r}_{B_{1}}^{0} \times \mathbf{u}_{1}\right] \\
\mathcal{F}_{a 2}=\left[\mathbf{u}_{2}, \mathbf{r}_{B_{2}}^{0} \times \mathbf{u}_{2}\right] \\
\mathcal{F}_{a 3}=\left[\mathbf{u}_{3}, \mathbf{r}_{B_{3}}^{0} \times \mathbf{u}_{3}\right] \\
\mathcal{W}_{a}=\operatorname{span}\left(\mathcal{F}_{a 1}, \mathcal{F}_{a 2}, \mathcal{F}_{a 3}\right)
\end{gathered}
$$

Due to the manipulator architecture, each leg applies one constraint force, which is perpendicular to the actuated prismatic joint and parallel to the axis $\mathbf{s}_{i}$ of the revolute 
joint, written as:

$$
\begin{gathered}
\mathcal{F}_{c 1}=\left[\mathbf{s}_{1}, \mathbf{r}_{B_{1}}^{0} \times \mathbf{s}_{1}\right] \\
\mathcal{F}_{c 2}=\left[\mathbf{s}_{2}, \mathbf{r}_{B_{2}}^{0} \times \mathbf{s}_{2}\right] \\
\mathcal{F}_{c 3}=\left[\mathbf{s}_{3}, \mathbf{r}_{B_{3}}^{0} \times \mathbf{s}_{3}\right] \\
\mathcal{W}_{c}=\operatorname{span}\left(\mathcal{F}_{c 1}, \mathcal{F}_{c 2}, \mathcal{F}_{c 3}\right)
\end{gathered}
$$

By collecting all components of the extended Jacobian matrix, we obtained:

$$
\mathbf{J}_{E}^{T}=\left[\begin{array}{llllll}
\mathcal{F}_{a 1} & \mathcal{F}_{a 2} & \mathcal{F}_{a 3} & \mathcal{F}_{c 1} & \mathcal{F}_{c 2} & \mathcal{F}_{c 3}
\end{array}\right]
$$

The degeneracy of matrix $\mathbf{J}_{E}$ indicates that the manipulator reaches a singularity configuration. We can observe the pose of the manipulator when $r_{1}=r_{2}=r_{3}=\sqrt{2} \mathrm{~m}$, the matrix $\mathbf{J}_{E}$ in this pose is rank deficient, while neither the constraint wrench system nor the actuation wrench system degenerates, i.e. $\operatorname{rank}\left(\mathbf{J}_{E}\right)=5, \operatorname{rank}\left(\mathcal{W}_{a}\right)=3$, and $\operatorname{rank}\left(\mathcal{W}_{c}\right)=3$. This means that the manipulator reaches an actuation singularity.

By examining the null space of the degenerate matrix $\mathbf{J}_{E}$, the uncontrolled motion (infinitesimal gain motion) of the moving platform can be obtained. This uncontrolled motion is characterized by a zero-pitch twist that is reciprocal to all constraint and actuation wrenches. It is denoted by $\mathbf{s}_{\lambda}$ and is described in Eq. (36). This singularity posture is depicted in Fig. 4, the uncontrolled motion of the moving platform is along the purple line.

$$
\mathbf{s}_{\lambda}^{T}=\left[\begin{array}{llllll}
1 & 1 & 1 & 0 & 0 & 0
\end{array}\right]
$$

\section{General Motion}

The set of eight constraint equations is written as a polynomial ideal $\mathcal{I}$ with variables $x_{0}, x_{1}, x_{2}, x_{3}, y_{0}, y_{1}, y_{2}, y_{3}$ over the coefficient ring $\mathbb{C}\left[h_{0}, h_{1}, r_{1}, r_{2}, r_{3}\right]$. 


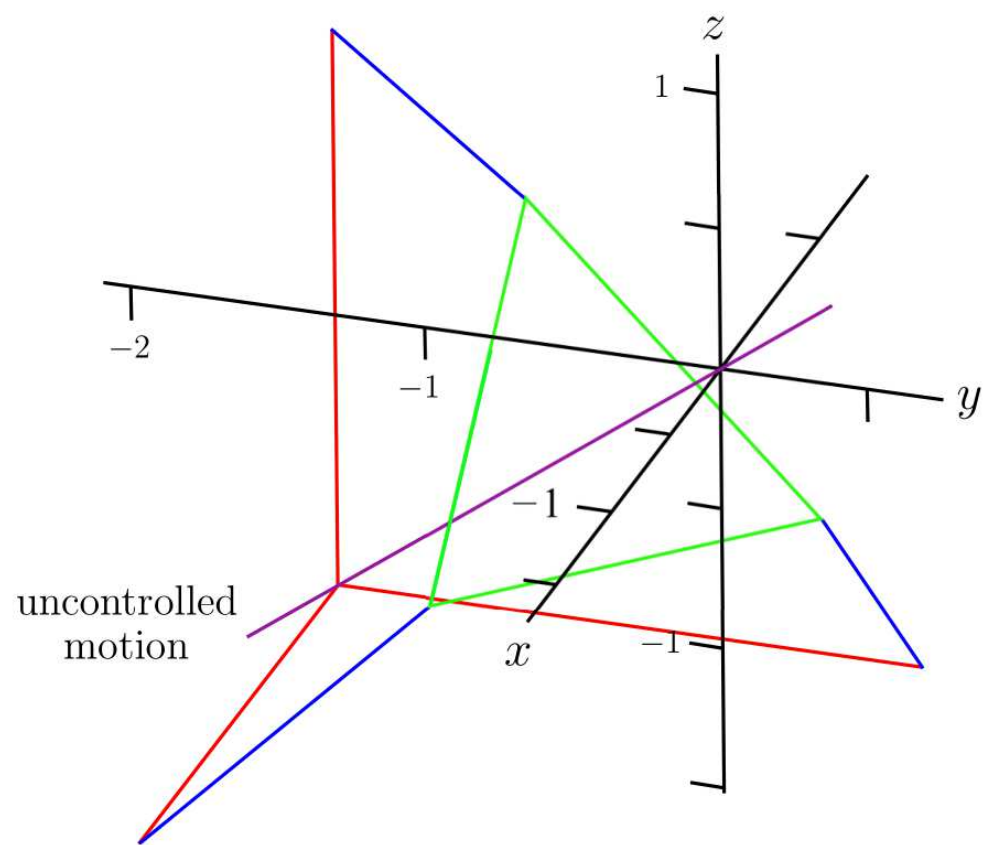

Figure 4: Singularity Pose at the Identity Condition.

$$
\mathcal{I}=<g_{1}, g_{2}, g_{3}, g_{4}, g_{5}, g_{6}, g_{7}, g_{8}>
$$

The general motion performed by the 3-RPS Cube parallel manipulator is characterized by solving the ideal $\mathcal{I}$. The equations $g_{1}, g_{2}, g_{3}, g_{4}, g_{5}, g_{6}, g_{7}$ from ideal $\mathcal{I}$ can be solved linearly for variables $y_{0}, y_{1}, y_{2}, y_{3}, R_{1}, R_{2}, R_{3}$ [12], $R_{i}$ being the square of the prismatic lengths, i.e., $R_{i}=r_{i}^{2}$, and $\delta=x_{0}^{2}+x_{1}^{2}+x_{2}^{2}+x_{3}^{2}$. Hence, the Study parameters become: 


$$
\begin{aligned}
& y_{0}=\frac{h_{1}\left(x_{1}^{2} x_{2}+x_{1}^{2} x_{3}+x_{1} x_{2}^{2}+x_{1} x_{3}^{2}+x_{2}^{2} x_{3}+x_{2} x_{3}^{2}\right)}{\delta} \\
& y_{1}=-\frac{h_{1}\left(x_{0}^{2} x_{2}-x_{0}^{2} x_{3}+x_{0} x_{2}^{2}+x_{0} x_{3}^{2}-x_{2}^{2} x_{3}+x_{2} x_{3}^{2}\right)}{\delta} \\
& y_{2}=\frac{h_{1}\left(x_{0}^{2} x_{1}-x_{0}^{2} x_{3}-x_{0} x_{1}^{2}-x_{0} x_{3}^{2}-x_{1}^{2} x_{3}+x_{1} x_{3}^{2}\right)}{\delta} \\
& y_{3}=-\frac{h_{1}\left(x_{0}^{2} x_{1}-x_{0}^{2} x_{2}+x_{0} x_{1}^{2}+x_{0} x_{2}^{2}-x_{1}^{2} x_{2}+x_{1} x_{2}^{2}\right)}{\delta}
\end{aligned}
$$

The terms $R_{i}{ }^{2}$ are also expressed in terms of $x_{0}, x_{1}, x_{2}, x_{3}$. The remaining Study parameters are still linked in equation $g_{8}: x_{0}^{2}+x_{1}^{2}+x_{2}^{2}+x_{3}^{2}-1=0$, which amounts to a hypersphere equation in space $\left(x_{0}, x_{1}, x_{2}, x_{3}\right)$. Accordingly, the transformation matrix is obtained. However, only the translational part of the transformation matrix depends on parameters $\left(x_{0}, x_{1}, x_{2}, x_{3}\right)$.

$$
\mathbf{M}_{T}=\left(\begin{array}{c}
2 h_{1}\left(x_{0} x_{2}-x_{0} x_{3}+x_{1} x_{2}+x_{1} x_{3}\right) \\
-2 h_{1}\left(x_{0} x_{1}-x_{0} x_{3}-x_{1} x_{2}-x_{2} x_{3}\right) \\
2 h_{1}\left(x_{0} x_{1}-x_{0} x_{2}+x_{1} x_{3}+x_{2} x_{3}\right)
\end{array}\right)
$$

This parametrization provides us with an interpretation of the general motion performed by the manipulator. The moving platform of the manipulator is capable of all orientations determined by $\left(x_{0}, x_{1}, x_{2}, x_{3}\right)$. The translational motion is coupled to the orientations via Eq. (39).

The position of any point in the moving platform $\left([1, x, y, z]^{T}\right)$ with respect to the fixed frame $\Sigma_{0}\left([1, X, Y, Z]^{T}\right)$ during the motion is determined by:

${ }^{2}$ The expressions are very lengthy and the reader may refer to
http://ww. irccyn.ec-nantes.fr/ caro/ASME_JMR/JMR_14_1262/Appendix_3RPSCube.pdf




$$
\begin{aligned}
\delta= & x_{0}^{2}+x_{1}^{2}+x_{2}^{2}+x_{3}^{2} \\
X= & \frac{1}{\delta}\left(\left(x_{0}^{2}+x_{1}^{2}-x_{2}^{2}-x_{3}^{2}\right) x+\left(-2 x_{0} x_{3}+2 x_{1} x_{2}\right) y+\left(2 x_{0} x_{2}+2 x_{1} x_{3}\right) z\right. \\
& \left.+2 h_{1}\left(x_{0} x_{2}-x_{0} x_{3}+x_{1} x_{2}+x_{1} x_{3}\right)\right) \\
Y= & \frac{1}{\delta}\left(\left(2 x_{0} x_{3}+2 x_{1} x_{2}\right) x+\left(x_{0}^{2}-x_{1}^{2}+x_{2}^{2}-x_{3}^{2}\right) y+\left(-2 x_{0} x_{1}+2 x_{2} x_{3}\right) z\right. \\
& \left.+2 h_{1}\left(-x_{0} x_{1}+x_{0} x_{3}+x_{1} x_{2}+x_{2} x_{3}\right)\right) \\
Z & \frac{1}{\delta}\left(\left(-2 x_{0} x_{2}+2 x_{1} x_{3}\right) x+\left(2 x_{0} x_{1}+2 x_{2} x_{3}\right) y+\left(x_{0}^{2}-x_{1}^{2}-x_{2}^{2}+x_{3}^{2}\right) z\right. \\
& \left.+2 h_{1}\left(x_{0} x_{1}-x_{0} x_{2}+x_{1} x_{3}+x_{2} x_{3}\right)\right)
\end{aligned}
$$

Let us consider a point $Q$ of coordinates $\mathbf{r}_{Q}^{1}=\left[1,-h_{1},-h_{1},-h_{1}\right]^{T}$, which is a special point in the cube of a moving frame $\Sigma_{1}$ as shown in Fig. 5. Then, its positions with respect to the fixed frame $\Sigma_{0}$ according to Eq. (40) are:

$$
\begin{aligned}
\delta & =x_{0}^{2}+x_{1}^{2}+x_{2}^{2}+x_{3}^{2} \\
X & =-h_{1}\left(x_{0}^{2}+x_{1}^{2}-x_{2}^{2}-x_{3}^{2}\right) / \delta \\
Y & =-h_{1}\left(x_{0}^{2}-x_{1}^{2}+x_{2}^{2}-x_{3}^{2}\right) / \delta \\
Z & =-h_{1}\left(x_{0}^{2}-x_{1}^{2}-x_{2}^{2}+x_{3}^{2}\right) / \delta
\end{aligned}
$$

The coordinates of point $Q$ depend on $\left(x_{0}, x_{1}, x_{2}, x_{3}\right)$. There are four possible positions corresponding to the three parameters (among four parameters $x_{i}, i=0,1,2,3$ ) are equal to zero. These corresponding positions of $Q$ are: 


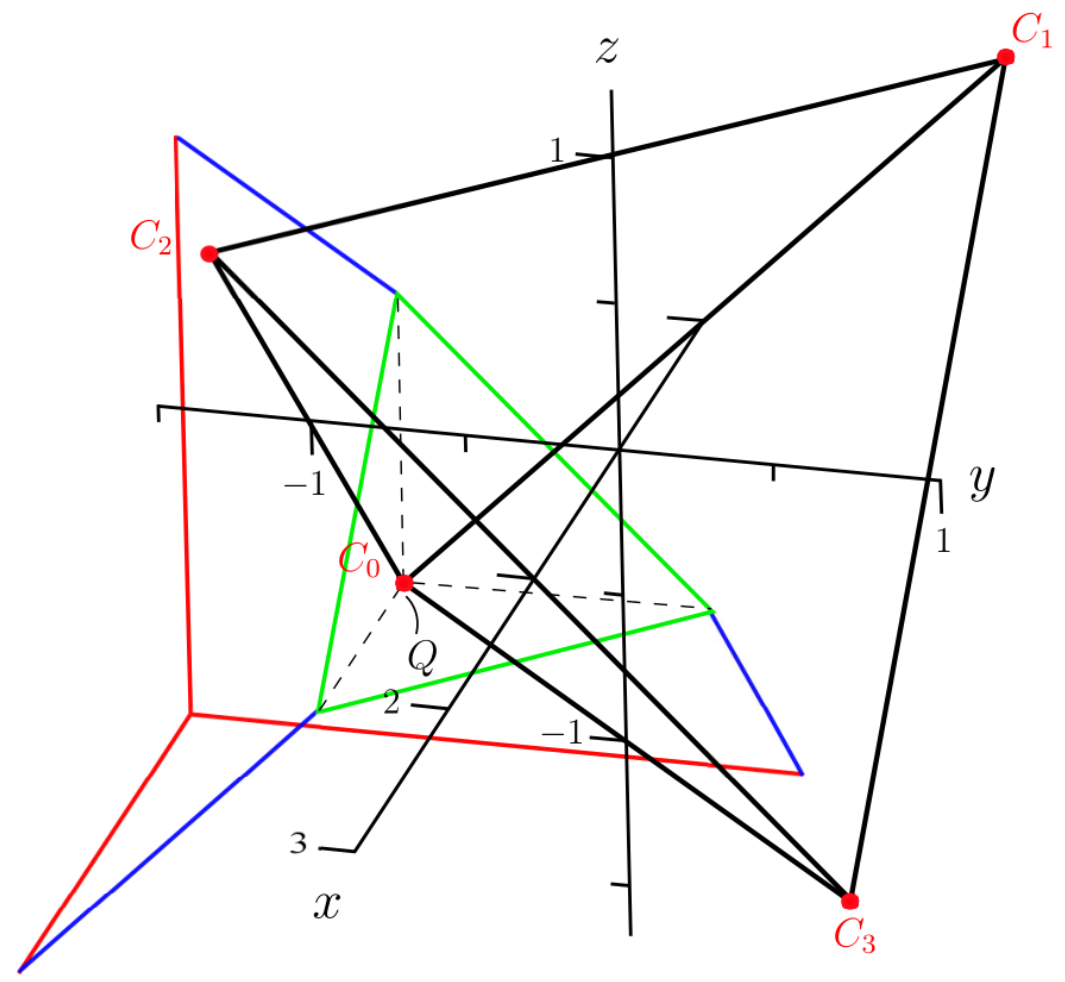

Figure 5: Tetrahedron $C$.

$$
\begin{aligned}
& x_{0}=1, x_{1}=x_{2}=x_{3}=0 \quad: \quad \mathbf{r}_{C_{0}}^{0}=\left[1,-h_{1},-h_{1},-h_{1}\right]^{T} \\
& x_{1}=1, x_{0}=x_{2}=x_{3}=0 \quad: \quad \mathbf{r}_{C_{1}}^{0}=\left[1,-h_{1}, h_{1}, h_{1}\right]^{T} \\
& x_{2}=1, x_{0}=x_{1}=x_{3}=0 \quad: \quad \mathbf{r}_{C_{2}}^{0}=\left[1, h_{1},-h_{1}, h_{1}\right]^{T} \\
& x_{3}=1, x_{0}=x_{1}=x_{2}=0 \quad: \quad \mathbf{r}_{C_{3}}^{0}=\left[1, h_{1}, h_{1},-h_{1}\right]^{T}
\end{aligned}
$$

$C_{0}, C_{1}, C_{2}$ and $C_{3}$ are the vertices of a tetrahedron $C$ as shown in Fig. 5. Those points correspond to the poses of the moving platform subjected to the actuation singularities. The uncontrolled motions of the moving platform are characterized by zero-pitch twists that intersect the geometric center of the moving platform and the corresponding vertices.

If two parameters are null, for instance $x_{2}=x_{3}=0$, the motion of point $Q$ will be determined by: 


$$
\begin{aligned}
& X=-h_{1} \\
& Y=-h_{1}\left(x_{0}^{2}-x_{1}^{2}\right) /\left(x_{0}^{2}+x_{1}^{2}\right) \\
& Z=-h_{1}\left(x_{0}^{2}-x_{1}^{2}\right) /\left(x_{0}^{2}+x_{1}^{2}\right)
\end{aligned}
$$

This means that point $Q$ moves along the edge $C_{0} C_{1}$, covering the closed interval between the two vertices. If only one parameter is zero, for instance if $x_{0}=0$, the point $Q$ will occupy the closed triangle $C_{1} C_{2} C_{3}$. Eventually, if none of the parameters is null, then point $Q$ will move inside the tetrahedron $C$.

Let us consider an arbitrary point $R$ in the moving platform such that:

$$
\left(x+h_{1}\right)\left(y+h_{1}\right)\left(z+h_{1}\right) \neq 0
$$

For example, take a point at the geometric center of the triangular-shaped platform, of coordinates $\mathbf{r}_{R}^{1}=\left[1,-\frac{2}{3} h_{1},-\frac{2}{3} h_{1},-\frac{2}{3} h_{1}\right]^{T}$. If any of the three parameters is zero, then the corresponding positions of point $R$ will become:

$$
\begin{array}{ll}
x_{0}=1, x_{1}=x_{2}=x_{3}=0 \quad: & \mathbf{r}_{D_{0}}^{0}=\left[1,-\frac{2}{3} h_{1},-\frac{2}{3} h_{1},-\frac{2}{3} h_{1}\right]^{T} \\
x_{1}=1, x_{0}=x_{2}=x_{3}=0 & : \quad \mathbf{r}_{D_{1}}^{0}=\left[1,-\frac{2}{3} h_{1}, \frac{2}{3} h_{1}, \frac{2}{3} h_{1}\right]^{T} \\
x_{2}=1, x_{0}=x_{1}=x_{3}=0 & : \quad \mathbf{r}_{D_{2}}^{0}=\left[1, \frac{2}{3} h_{1},-\frac{2}{3} h_{1}, \frac{2}{3} h_{1}\right]^{T} \\
x_{3}=1, x_{0}=x_{1}=x_{2}=0 & : \quad \mathbf{r}_{D_{3}}^{0}=\left[1, \frac{2}{3} h_{1}, \frac{2}{3} h_{1},-\frac{2}{3} h_{1}\right]^{T}
\end{array}
$$

$D_{0}, D_{1}, D_{2}$ and $D_{3}$ are the vertices of a pseudo-tetrahedron $D$ as shown in Fig. 6 and it was verified that these vertices amount to the singularities of the 3-RPS Cube manipulator. If two parameters are equal to zero, for instance $x_{2}=x_{3}=0$, the point $Q$ will move along the edge $C_{0} C_{1}$, while the path of point $R$ will be given by: 


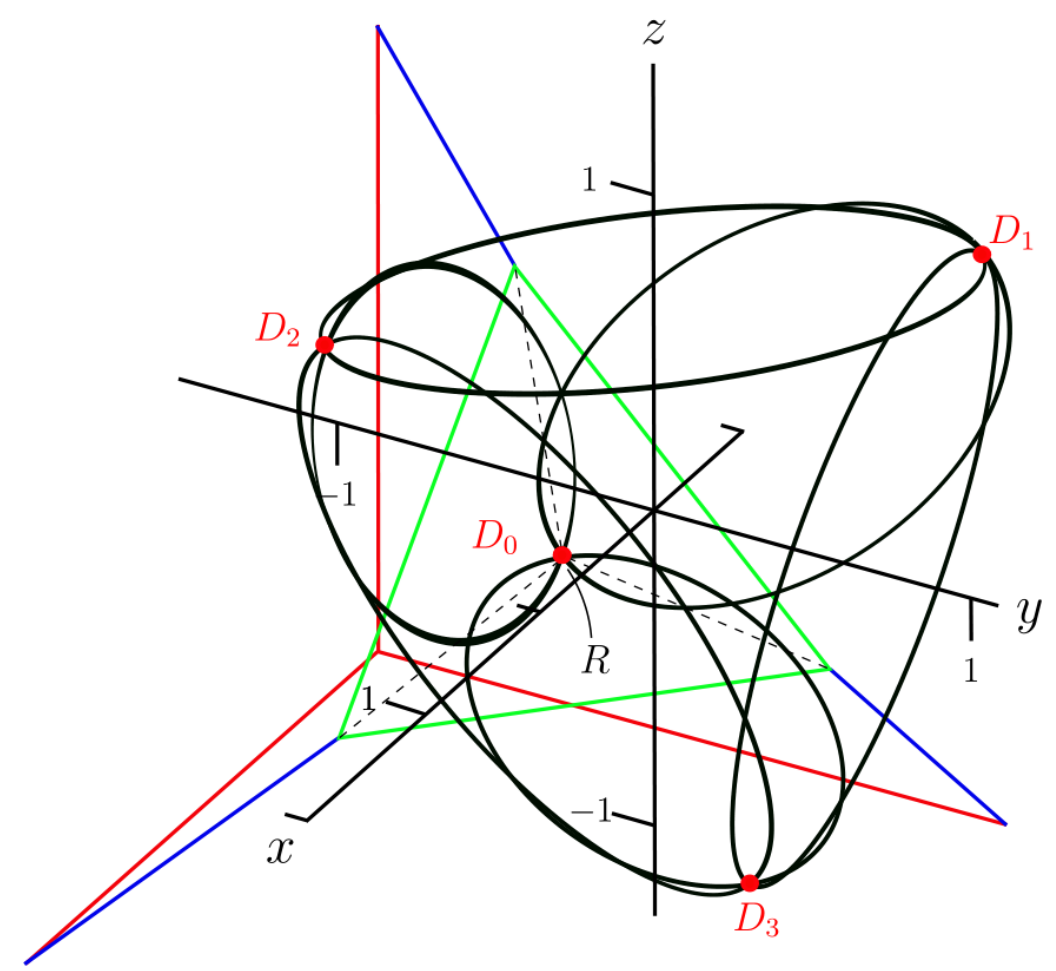

Figure 6: Pseudo-tetrahedron $D$.

$$
\begin{aligned}
& X=-\frac{2}{3} h_{1} \\
& Y=-\frac{2}{3} \frac{h_{1}\left(x_{0}^{2}+x_{0} x_{1}-x_{1}^{2}\right)}{x_{0}^{2}+x_{1}^{2}} \\
& Z=-\frac{2}{3} \frac{h_{1}\left(x_{0}^{2}-x_{0} x_{1}-x_{1}^{2}\right)}{x_{0}^{2}+x_{1}^{2}}
\end{aligned}
$$

This represents an ellipse $\mathbf{e}_{01}$ that passes through the vertices $D_{0}$ and $D_{1}$ and lies in the plane $X=-\frac{2}{3} h_{1}$. Accordingly, the four vertices of the pseudo-tetrahedron $D$ are joined by six ellipses, as shown in Fig. 6 .

When only one parameter is equal to zero, for instance $x_{0}=0$, the trajectory of point $R$ will follow a particular surface, called the Steiner surface $\mathbf{F}_{0}{ }^{3}$. It passes through the

\footnotetext{
${ }^{3}$ The motion animation of point $R$ that is bounded by the Steiner surface, is shown in: http://www.irccyn.ec-nantes.fr/ caro/ASME_JMR/JMR_14_1262/animation_steiner.gif
} 


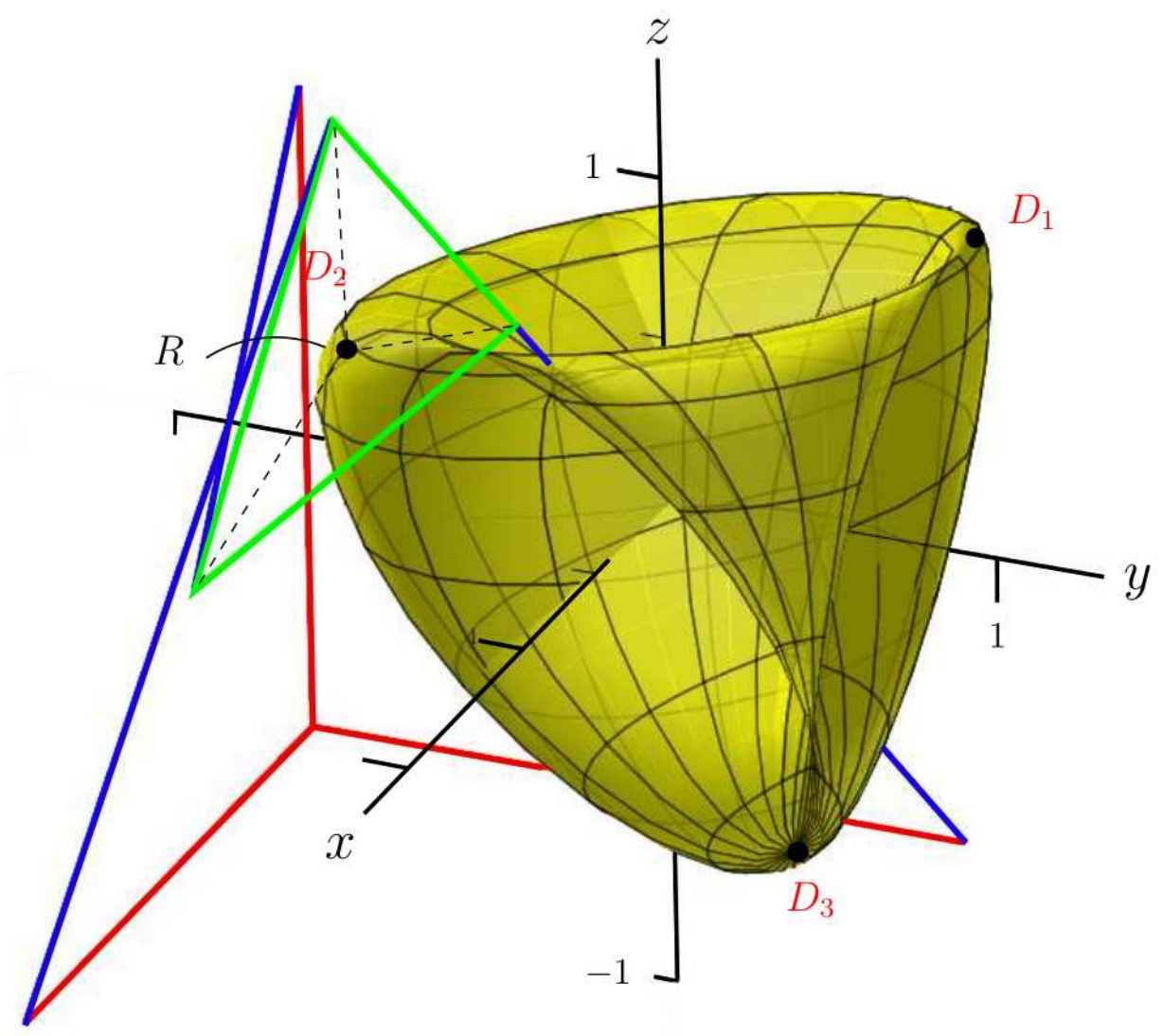

Figure 7: Steiner Surface $\mathbf{F}_{0}$.

vertices $D_{1}, D_{2}, D_{3}$ and the ellipses $\mathbf{e}_{12}, \mathbf{e}_{13}, \mathbf{e}_{23}$, which is illustrated in yellow in Fig. 7 . Then the expressions of the trajectory of point $R$ are given by:

$$
\begin{aligned}
& X=-\frac{2}{3} \frac{h_{1}\left(x_{1}^{2}-x_{1} x_{2}-x_{1} x_{3}-x_{2}^{2}-x_{3}^{2}\right)}{\left(x_{1}^{2}+x_{2}^{2}+x_{3}^{2}\right)} \\
& Y=\frac{2}{3} \frac{h_{1}\left(x_{1}^{2}+x_{1} x_{2}-x_{2}^{2}+x_{2} x_{3}+x_{3}^{2}\right)}{\left(x_{1}^{2}+x_{2}^{2}+x_{3}^{2}\right)} \\
& Z=\frac{2}{3} \frac{h_{1}\left(x_{1}^{2}+x_{1} x_{3}+x_{2}^{2}+x_{2} x_{3}-x_{3}^{2}\right)}{\left(x_{1}^{2}+x_{2}^{2}+x_{3}^{2}\right)}
\end{aligned}
$$

Therefore, the trajectory of an arbitrary point of the moving platform forms the shape of pseudo-tetrahedron $D$ and contains four vertices $D_{i}(i=0,1,2,3)$. These vertices are 
joined by six ellipses and any three of the vertices are linked by a Steiner surface $\mathbf{F}_{j}$ $(j=0,1,2,3)$. Any two Steiner surfaces $\left(\mathbf{F}_{i}\right.$ and $\left.\mathbf{F}_{j}\right)$ share one ellipse $\mathbf{e}_{i j}$ in common.

Let us analyse the motion of a special point $S$ that does not fulfill Eq. (44). For instance, the point $S$ is at one vertex of the triangular-shaped platform, $B_{3}$ (Fig. 1). If three parameters (among four parameters $x_{i}, i=0,1,2,3$ ) are equal to zero, the positions of the point $S$ are determined by:

$$
\begin{array}{lll}
x_{0}=1, x_{1}=x_{2}=x_{3}=0 \quad: & \mathbf{r}_{E_{0}}^{0}=\left[1,-h_{1},-h_{1}, 0\right]^{T} \\
x_{1}=1, x_{0}=x_{2}=x_{3}=0 \quad: & \mathbf{r}_{E_{1}}^{0}=\left[1,-h_{1}, h_{1}, 0\right]^{T} \\
x_{2}=1, x_{0}=x_{1}=x_{3}=0 \quad: & \mathbf{r}_{E_{2}}^{0}=\left[1, h_{1},-h_{1}, 0\right]^{T} \\
x_{3}=1, x_{0}=x_{1}=x_{2}=0 \quad: & \mathbf{r}_{E_{3}}^{0}=\left[1, h_{1}, h_{1}, 0\right]^{T}
\end{array}
$$

Those points are coplanar and are the vertices of a rectangle as shown in Fig. 8. If two parameters are zero, for example $x_{2}=x_{3}=0$, the path of point $S$ is along the edge $E_{0} E_{1}$. Accordingly, in a general configuration the point $S$ always moves in the plane $Z=0$.

Another special point which does not fulfill Eq. (44) is the origin of the moving frame $P$. According to Eq. (40), the positions of point $P$ are given by:

$$
\begin{aligned}
\delta & =x_{0}^{2}+x_{1}^{2}+x_{2}^{2}+x_{3}^{2} \\
X & =\frac{1}{\delta} 2 h_{1}\left(x_{0} x_{2}-x_{0} x_{3}+x_{1} x_{2}+x_{1} x_{3}\right) \\
Y & =\frac{1}{\delta} 2 h_{1}\left(-x_{0} x_{1}+x_{0} x_{3}+x_{1} x_{2}+x_{2} x_{3}\right) \\
Z & =\frac{1}{\delta} 2 h_{1}\left(x_{0} x_{1}-x_{0} x_{2}+x_{1} x_{3}+x_{2} x_{3}\right)
\end{aligned}
$$

If three parameters (among four parameters $x_{i}, i=0,1,2,3$ ) are equal to zero, the positions of the point $P$ will be always coincident with the origin of the fixed frame $O$. 


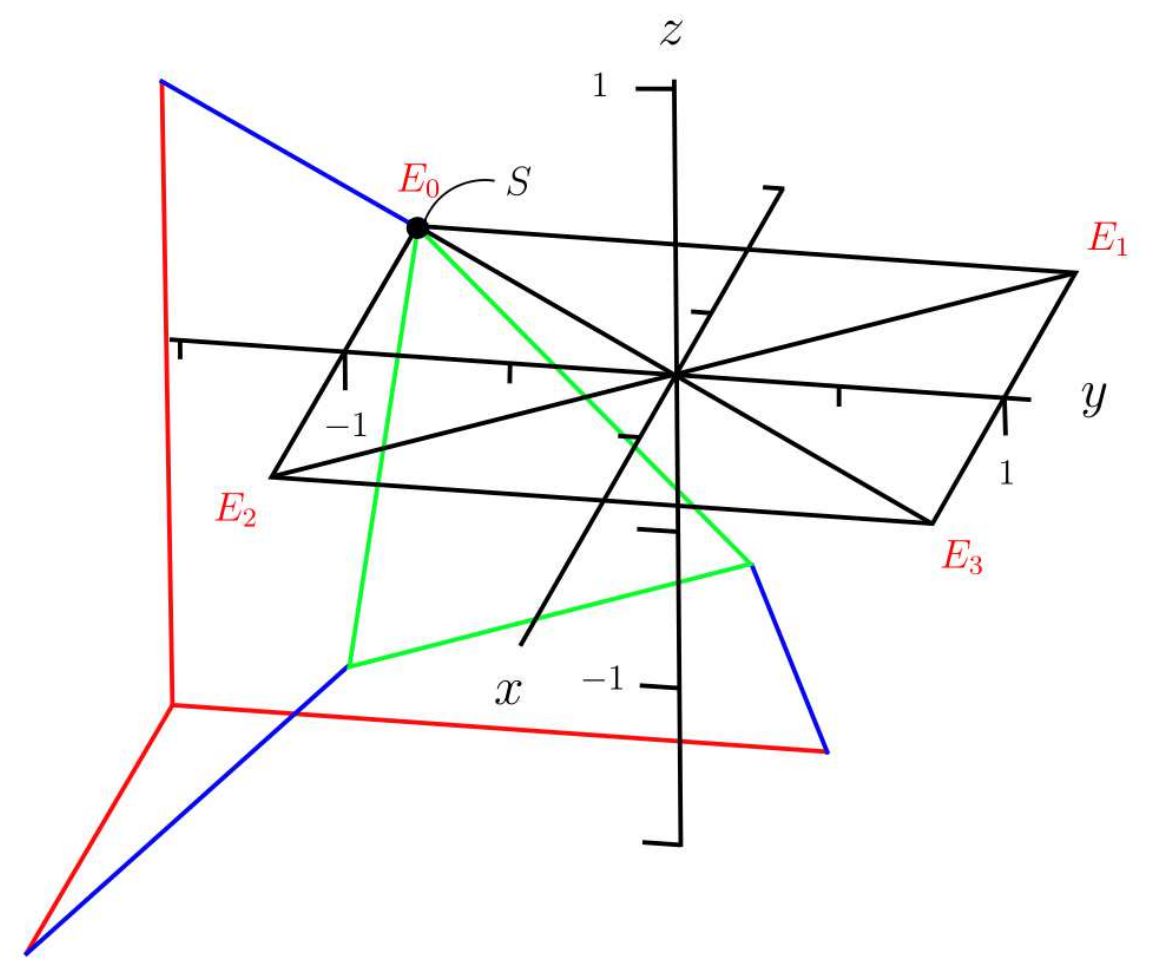

Figure 8: Rectangle E. 


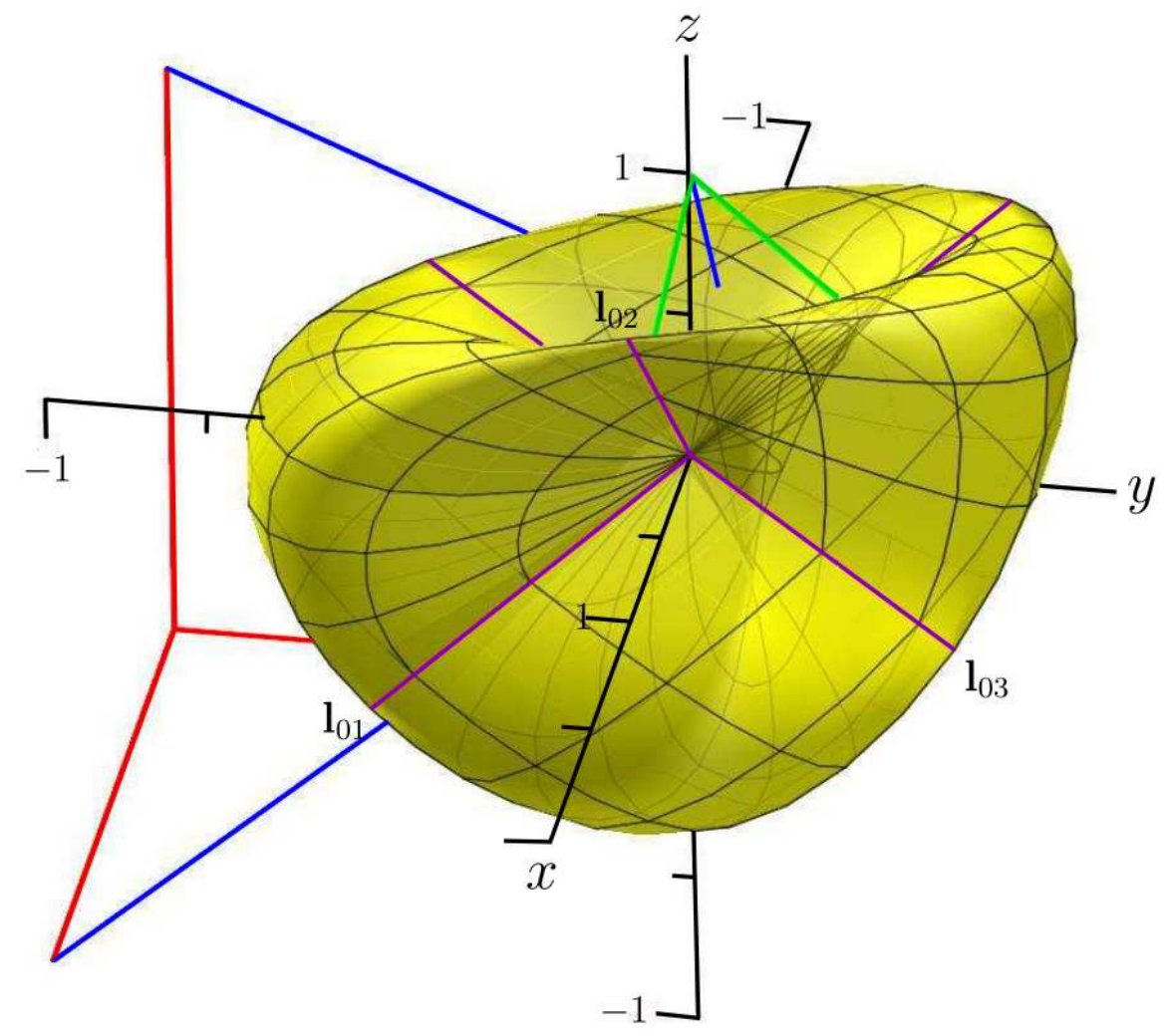

Figure 9: Steiner Surface $\mathbf{G}_{0}$.

If two parameters are zero, for example $x_{0}=x_{1}=0$, the point $P$ will move along a line $\mathbf{l}_{01}$ that passes through the point $O$ and lies on the plane $X=0$. Its endpoints are $[0,-1,-1]^{T}$ and $[0,1,1]^{T}$. For $x_{2}=x_{3}=0$, the point $P$ moves along a line $\mathbf{l}_{23}$ that also lies on the plane $X=0$.

If only one parameter is equal to zero, for example $x_{0}=0$, the point $P$ moves on the Steiner surface $\mathbf{G}_{0}$. Unlike the Steiner surface $\mathbf{F}_{0}$ generated by the motion of point $R$, the Steiner surface $\mathbf{G}_{0}$ is elongated as illustrated in yellow in Fig. 9. The Steiner surface $\mathbf{G}_{0}$ contains the point $O$ and three lines $\mathbf{l}_{01}, \mathbf{l}_{02}, \mathbf{l}_{03}$, shown as purple lines in Fig. 9. 


\section{Vertical Darboux Motion}

The condition for the manipulator to generate the VDM is that all prismatic lengths are equal, i.e., $r_{1}=r_{2}=r_{3}$. By solving the direct kinematics of the manipulator with the same prismatic lengths, the Study parameters obtained to perform the VDM yield $x_{1}=x_{2}=x_{3}$ and $y_{1}=y_{2}=y_{3}$. By substituting those values into the ideal $\mathcal{I}$, the set of eight constraint equations becomes:

$$
\begin{gathered}
\mathcal{I}:\left\{-x_{0} y_{1}-2 x_{1}^{2}+x_{1} y_{0}=0,-x_{0} y_{1}-2 x_{1}^{2}+x_{1} y_{0}=0,-x_{0} y_{1}-2 x_{1}^{2}+x_{1} y_{0}=0,\right. \\
-R_{1} x_{0}^{2}-3 R_{1} x_{1}^{2}+2 x_{0}^{2}-8 x_{0} y_{1}+22 x_{1}^{2}+8 x_{1} y_{0}+4 y_{0}^{2}+12 y_{1}^{2}=0, \\
-R_{2} x_{0}^{2}-3 R_{2} x_{1}^{2}+2 x_{0}^{2}-8 x_{0} y_{1}+22 x_{1}^{2}+8 x_{1} y_{0}+4 y_{0}^{2}+12 y_{1}^{2}=0, \\
-R_{3} x_{0}^{2}-3 R_{3} x_{1}^{2}+2 x_{0}^{2}-8 x_{0} y_{1}+22 x_{1}^{2}+8 x_{1} y_{0}+4 y_{0}^{2}+12 y_{1}^{2}=0, \\
\left.y_{0} x_{0}+3 y_{1} x_{1}=0, x_{0}^{2}+3 x_{1}^{2}-1=0\right\}
\end{gathered}
$$

It follows from Eq. (50) that the first three constraint equations are the same. Likewise, the next three equations are identical. Mathematically, one has to find the case of 1-dof motion, as known as cylindrical motion, with one parameter that describes the VDM. Equation (50) can be solved linearly for the variables $R_{i}, y_{0}, y_{1}$ in terms of $x_{0}, x_{1}$, as follows:

$$
\begin{aligned}
& y_{0}=\frac{6 x_{1}^{3}}{\left(x_{0}^{2}+3 x_{1}^{2}\right)}, \quad y_{1}=-\frac{2 x_{0} x_{1}^{2}}{\left(x_{0}^{2}+3 x_{1}^{2}\right)} \\
& R_{1}=R_{2}=R_{3}=-\frac{\left(-2 x_{0}^{4}-44 x_{0}^{2} x_{1}^{2}-162 x_{1}^{4}\right)}{\left(x_{0}^{4}+6 x_{0}^{2} x_{1}^{2}+9 x_{1}^{4}\right)}
\end{aligned}
$$

From Eq. (51), it is apparent that the manipulator can perform the VDM if and only if all prismatic lengths are the same. The remaining Study parameters $x_{0}$ and $x_{1}$ are still 
linked to the eighth equation in $x_{0}^{2}+3 x_{1}^{2}-1=0$, which is simply an ellipse equation in the space $x_{0}$ and $x_{1}$. This ellipse equation can be parametrized by $x_{0}=\cos (u)$ and $x_{1}=\frac{1}{3} \sin (u) \sqrt{3}$.

As a result, the workspace of the manipulator performing the VDM is parametrized by the parameter $u$. Hence, the Study parameters are expressed as:

$$
\begin{array}{rrr}
x_{0}=\mathrm{c}(u) \quad x_{1}=\frac{1}{3} \mathrm{~s}(u) \sqrt{3} \quad y_{0}=\frac{2}{3} \mathrm{~s}(u)^{3} \sqrt{3} \quad y_{1}=-\frac{2}{3} \mathrm{c}(u) \mathrm{s}(u)^{2} \\
x_{2}=\frac{1}{3} \mathrm{~s}(u) \sqrt{3} \quad x_{3}=\frac{1}{3} \mathrm{~s}(u) \sqrt{3} \quad y_{2}=-\frac{2}{3} \mathrm{c}(u) \mathrm{s}(u)^{2} \quad y_{3}=-\frac{2}{3} \mathrm{c}(u) \mathrm{s}(u)^{2}
\end{array}
$$

where $\mathrm{s}(u)=\sin (u), \mathrm{c}(u)=\cos (u)$.

Therefore, the possible poses of the moving platform can be expressed by the following transformation matrix:

$$
\mathbf{T}=\left[\begin{array}{cccc}
1 & 0 & 0 & 0 \\
a & \frac{4}{3} \mathrm{c}(u)^{2}-\frac{1}{3} & -\frac{2}{3} \mathrm{~s}(u)(\mathrm{c}(u) \sqrt{3}-\mathrm{s}(u)) & -\frac{2}{3} \mathrm{~s}(u)(\mathrm{c}(u) \sqrt{3}-\mathrm{s}(u)) \\
a & -\frac{2}{3} \mathrm{~s}(u)(\mathrm{c}(u) \sqrt{3}-\mathrm{s}(u)) & \frac{4}{3} \mathrm{c}(u)^{2}-\frac{1}{3} & -\frac{2}{3} \mathrm{~s}(u)(\mathrm{c}(u) \sqrt{3}-\mathrm{s}(u)) \\
a & -\frac{2}{3} \mathrm{~s}(u)(\mathrm{c}(u) \sqrt{3}-\mathrm{s}(u)) & -\frac{2}{3} \mathrm{~s}(u)(\mathrm{c}(u) \sqrt{3}-\mathrm{s}(u)) & \frac{4}{3} \mathrm{c}(u)^{2}-\frac{1}{3}
\end{array}\right]
$$

where $a=\frac{4}{3} \sin (u)^{2}$.

\subsection{Trajectory of the moving platform performing the Ver- tical Darboux Motion}

Let us consider the point $B_{1}$ moving in the plane $X=0$ and the geometric center $R$ of the moving platform as shown in Fig. 1. The paths followed by those two points are obtained by setting $u=-\frac{\pi}{2} \ldots \frac{\pi}{2}$ by using the transformation matrix $\mathbf{T}$ defined in Eq. (53).

It appears that those two paths are different as shown in Fig. $10^{4}$. Point $R$ moves

${ }^{4}$ The animation of the trajectories is shown in: 


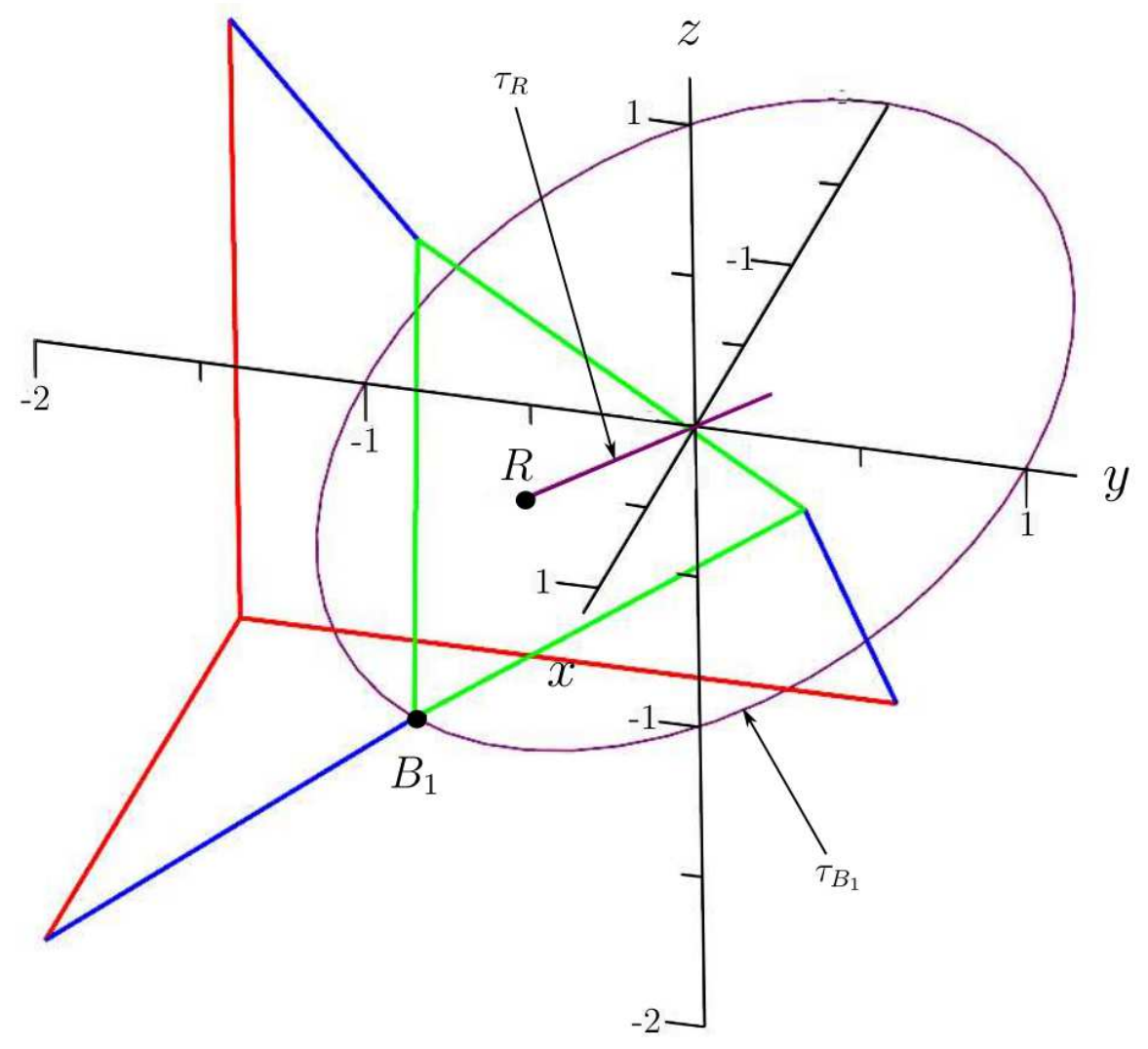

Figure 10: Trajectories of points $B_{1}$ and $R$.

along a straight line denoted as $\tau_{R}$, whereas point $B_{1}$ moves along a planar ellipse $\tau_{B_{1}}$ that is parallel to the plane $X=0$.

Let us take all segments joining point $B_{1}$ to any point of segment $B_{2} B_{3}$ and plot the paths of all points on those segments. All those paths are planar ellipses, except the path followed by point $R$. Accordingly, the set of all paths forms a ruled surface called Right-conoid surface, which is illustrated in yellow in Fig. $11^{5}$.

This type of ruled surfaces is generated by moving a straight line such that it intersects perpendicularly a fixed straight line, called the axis of the Right-conoid surface. The fixed straight line followed by point $R$ is the axis of the Right-conoid surface.

http://www.irccyn.ec-nantes.fr/ caro/ASME_JMR/JMR_14_1262/animation_trajectories.gif

${ }^{5}$ The animation of the right-conoid surface is shown in:

http://www.irccyn.ec-nantes.fr/ caro/ASME_JMR/JMR_14_1262/animation_rightconoid.gif 


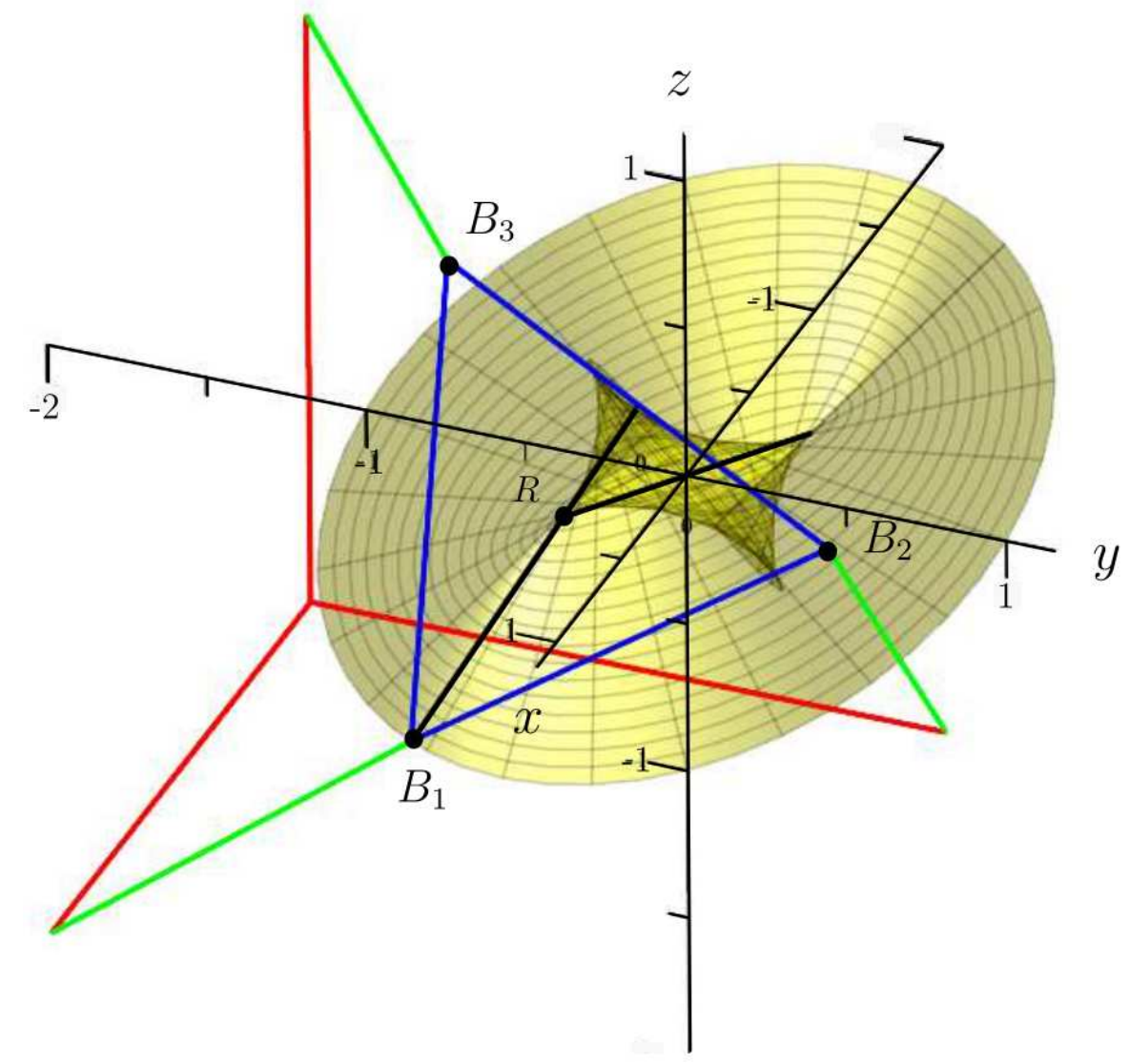

Figure 11: Right-conoid Surface of the VDM.

\subsection{Axodes of the manipulator performing the Vertical Dar- boux Motion}

Having the parametrization of the VDM performed by the 3-RPS Cube parallel manipulator in terms of Study parameters, it is relatively easy to compute the ISA. The possible poses of the moving platform as functions of time in this special motion only allow the orientations that are given by one parameter $u$. The ISA are obtained from the entries of the velocity operator:

$$
\mathbf{A}=\dot{\mathbf{T}} \mathbf{T}^{-1}
$$

By setting $u=t$, matrix A becomes: 


$$
\mathbf{A}=\left[\begin{array}{cccc}
0 & 0 & 0 & 0 \\
\frac{8}{3} c(t) \mathrm{s}(t) & 0 & -\frac{2}{3} \sqrt{3} & \frac{2}{3} \sqrt{3} \\
\frac{8}{3} \mathrm{c}(t) \mathrm{s}(t) & \frac{2}{3} \sqrt{3} & 0 & -\frac{2}{3} \sqrt{3} \\
\frac{8}{3} \mathrm{c}(t) \mathrm{s}(t) & -\frac{2}{3} \sqrt{3} & \frac{2}{3} \sqrt{3} & 0
\end{array}\right]
$$

The instantaneous screw axis of the moving platform is obtained from the components of matrix $\mathbf{A}$ as explained in [13], after normalization:

$$
I S A=\left[\begin{array}{llllll}
\frac{1}{\sqrt{3}} & \frac{1}{\sqrt{3}} & \frac{1}{\sqrt{3}} & \frac{4}{3} \mathrm{c}(t) \mathrm{s}(t) & \frac{4}{3} \mathrm{c}(t) \mathrm{s}(t) & \frac{4}{3} \mathrm{c}(t) \mathrm{s}(t)
\end{array}\right]^{T}
$$

All twists of the manipulator are collinear. As a consequence, the fixed axode generated by the ISA is a straight line of unit vector $[1 / \sqrt{3}, 1 / \sqrt{3}, 1 / \sqrt{3}]^{T}$. In the moving coordinate frame, the moving axode corresponding to this motion is congruent with the fixed axode as depicted in Fig. 12. However, the moving axode does not appear clearly as it is congruent with the fixed axode. Indeed, the moving axode internally slides and rolls onto the fixed axode.

\section{Conclusions}

In this paper, an algebraic geometry method was applied to analyse the kinematics and the operation mode of the 3-RPS Cube manipulator. Primary decomposition of an ideal of eight constraint equations revealed that the manipulator has only one general operation mode. In this operation mode, the direct kinematics was solved and the number of solutions was obtained for arbitrary values of design parameters and joint variables. The singularity conditions were computed and represented in the joint space. It turns out that the manipulator reaches the singularity when the moving frame coincides with the fixed frame and all joint variables are equal. The uncontrolled motion of the moving platform in this singularity configuration was investigated and geometrically interpreted. 


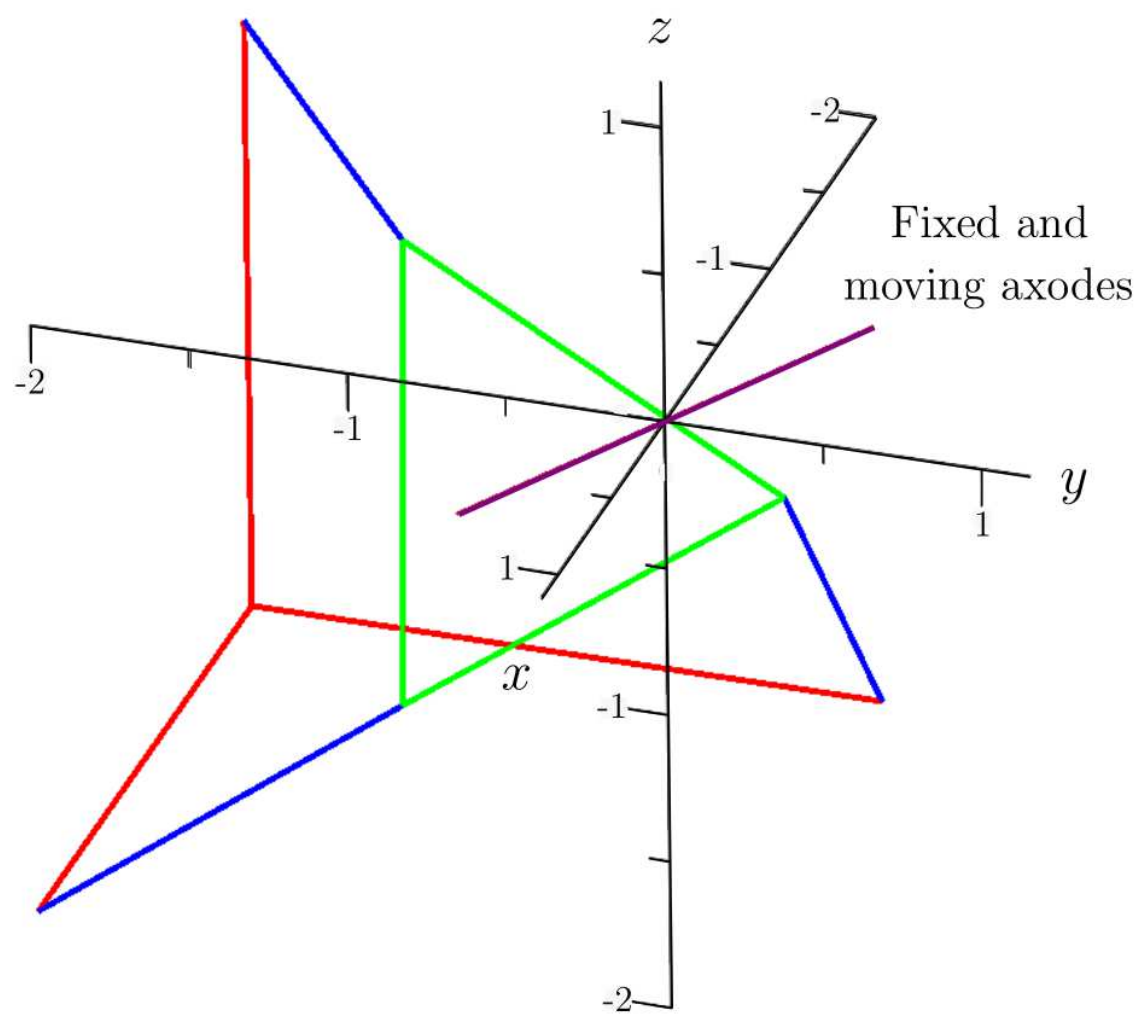

Figure 12: ISA Axodes of VDM.

The 3-dof motion of the 3-RPS Cube manipulator was discussed in this paper. The trajectory of each point in the moving platform, except the vertices of the moving platform, in general belongs to a special surface named the Steiner surface. The vertices of the triangular-shaped platform always move in the planes of a trihedron. By keeping all leg parameters the same, the 3-RPS Cube manipulator is able to generate the Vertical Darboux Motion (VDM). In this VDM the moving platform rotates about an axis and translates along the same direction. The paths followed by all points belonging to the moving platform, with the exception of its geometric center, are ellipses that form a ruled surface called a right-conoid surface. The geometric center of the moving platform moves along a straight line. Finally, since the 3-RPS Cube has only one operation mode, the general motion and the VDM occur inside the same operation mode. The investigation of collisions for workspace analysis or trajectory planning purposes will be the subject of 
future work.

\section{Acknowledgments}

The authors would like to acknowledge the support of the Österreichischer Austauschdienst/OeAD, the French Ministry for Foreign Affairs (MAEE) and the French Ministry for Higher Education and Research (MESR) (Project PHC AMADEUS). Moreover, Prof. Manfred Husty acknowledges the support of FWF grant P 23832-N13, Algebraic Methods in Collision Detection and Path Planning.

\section{References}

[1] Z. Huang and Y. Fang, "Motion Characteristics and Rotational Axis Analysis of Three DOF Parallel Robot Mechanisms," 1995 IEEE International Conference on Systems, Man and Cybernetics. Intelligent Systems for the 21st Century, vol. 1, no. 1, pp. $67-71,1995$.

[2] Z. Chen, W.-A. Cao, and Z. Huang, "Type Synthesis of 3-DOF Rotational Parallel Mechanisms With No Intersecting Axes," Proceedings of the ASME 2012 International Design Engineering Technical Conferences and Computers $\mathcal{E}$ Information in Engineering Conference, United States, 2012.

[3] Z. Huang and Y. Fang, "Kinematic Characteristics Analysis of 3 DOF in-Parallel Actuated Pyramid Mechanism," Mechanism and Machine Theory, vol. 31, no. 8, pp. 1009-1018, 1996.

[4] Z. Huang and J. Wang, "Identification of Principal Screws of 3-DOF Parallel Manipulators by Quadric Degeneration," Mechanism and Machine Theory, vol. 36, pp. 893$911,2001$.

[5] Z. Huang, J. Wang, and Y. Fang, "Analysis of Instantaneous Motions of Deficient Rank 3-RPS Parallel Manipulators," Mechanism and Machine Theory, vol. 37, pp. 229-240, 2002. 
[6] Z. Huang, D. Mu, and D. Zeng, "The Screw Motion Simulation on 3-RPS Parallel Pyramid Mechanism," 2007 International Conference on Mechatronics and Automation, pp. 2860-2864, Aug. 2007.

[7] C.-C. Lee and J. M. Hervé, "On the Vertical Darboux Motion," In: Springer (ed) Latest Advance in Robot Kinematics, pp. 99-106, 2012.

[8] Z. Huang, Z. Chen, J. Liu, and S. Liu, "A 3DOF Rotational Parallel Manipulator Without Intersecting Axes," Journal of Mechanisms and Robotics, vol. 3, no. 2, pp. 1-8, 2011.

[9] Z. Chen, H. Ding, W. Cao, and Z. Huang, "Axodes Analysis of the Multi DOF Parallel Mechanisms and Parasitic Motion," Proceedings of the ASME 2013 International Design Engineering Technical Conferences and Computers $\mathcal{E}$ Information in Engineering Conference, United States, 2013.

[10] D. R. Walter, M. L. Husty, and M. Pfurner, "A Complete Kinematic Analysis of the SNU 3-UPU Parallel Robot," Contemporary Mathematics, vol. 496, pp. 331-346, 2009.

[11] D. R. Walter and M. L. Husty, "Kinematic Analysis of the TSAI-3UPU Parallel Manipulator Using Algebraic Methods," 13th World Congress in Mechanism and Machine Science, vol. 1, pp. 19-25, 2011.

[12] J. Schadlbauer, D. R. Walter, and M. L. Husty, "A Complete Kinematic Analysis of the 3-RPS Parallel Manipulator," 15th National Conference on Machines and Mechanisms, pp. 1-10, 2011.

[13] J. Schadlbauer, L. Nurahmi, M. Husty, P. Wenger, and S. Caro, "Operation Modes in Lower Mobility Parallel Manipulators," In Second Conference in Interdisciplinary Applications in Kinematics, pp. 3-10, 2013.

[14] J. Schadlbauer, D. R. Walter, and M. L. Husty, "The 3-RPS parallel manipulator from an algebraic viewpoint," Mechanism and Machine Theory, vol. 75, pp. 161-176, May 2014. 
[15] O. Bottema and B. Roth, Theoretical Kinematics. New York: Dover Publications, 1990.

[16] S. A. Joshi and L.-W. Tsai, "Jacobian Analysis of Limited-DOF Parallel Manipulators," Journal of Mechanical Design, vol. 124, no. 2, pp. 254-258, 2002.

[17] S. Amine, S. Caro, P. Wenger, and D. Kanaan, "Singularity Analysis of the H4 Robot Using Grassmann-Cayley Algebra," Robotica, vol. 30, no. 7, pp. 1109-1118, 2012.

[18] S. Amine, Lower-Mobility Parallel Manipulators: Geometrical Analysis, Singularities and Conceptual Design. PhD thesis, 2011.

[19] S. Amine, M. T. Masouleh, S. Caro, P. Wenger, and C. Gosselin, "Singularity analysis of 3T2R parallel mechanisms using Grassmann Cayley algebra and Grassmann geometry," Mechanism and Machine Theory, vol. 52, pp. 326-340, June 2012.

[20] S. Amine, L. Nurahmi, P. Wenger, and S. Caro, "Conceptual Design of Schonflies Motion Generators Based on the Wrench Graph," Proceedings of the ASME 2013 International Design Engineering Technical Conferences and Computers 8 Information in Engineering Conference, United States, 2013.

[21] S. Amine, M. T. Masouleh, S. Caro, P. Wenger, and C. Gosselin, "Singularity Conditions of 3T1R Parallel Manipulators With Identical Limb Structures," ASME Journal of Mechanism and Robotics, vol. 4, pp. 1-11, Feb. 2012.

[22] H. Pottmann and J. Wallner, Computational Line Geometry. Springer Verlag Berlin, 2001.

\section{List of Figures}

1 The 3 -RPS Cube Parallel Manipulator. . . . . . . . . . . . 6

2 Solutions of the Direct Kinematics. . . . . . . . . . . . . . . . . . . 16

$3 \quad$ Singularity Surface of $\mathcal{L}_{1} \ldots \ldots \ldots \ldots \ldots \ldots \ldots$

4 Singularity Pose at the Identity Condition. . . . . . . . . . . . . . . . 20

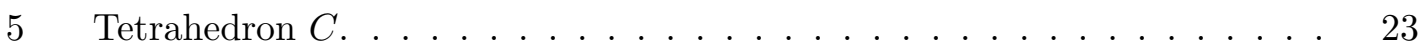




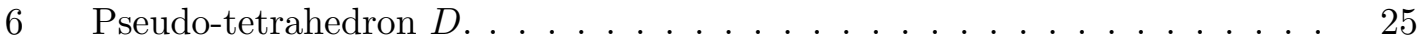

$7 \quad$ Steiner Surface $\mathbf{F}_{0} \ldots \ldots \ldots \ldots \ldots \ldots$

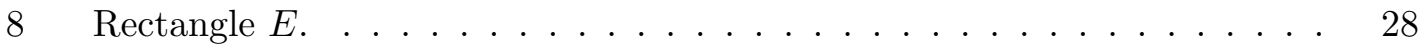

$9 \quad$ Steiner Surface $\mathbf{G}_{0} \ldots \ldots \ldots \ldots \ldots$

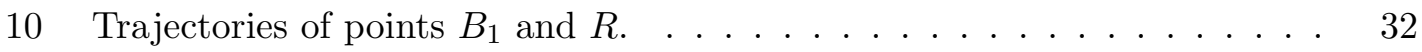

11 Right-conoid Surface of the VDM. . . . . . . . . . . . . . . . . . 33

12 ISA Axodes of VDM. . . . . . . . . . . . . . . . . . 35 